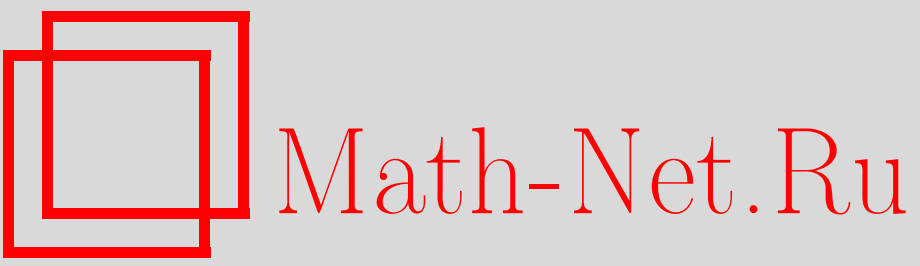

А. В. Жибер, В. В. Соколов, Точно интегрируемые гиперболические уравнения лиувиллевского типа, УМН, 2001, том 56, выпуск 1, 63-106

DOI: https://doi.org/10.4213/rm357

Использование Общероссийского математического портала Math-Net.Ru подразумевает, что вы прочитали и согласны с пользовательским соглашением

http://www.mathnet.ru/rus/agreement

Параметры загрузки:

IP: 52.90 .164 .192

26 апреля 2023 г., 13:08:46 


\title{
ТОЧНО ИНТЕГРИРУЕМЫЕ ГИПЕРБОЛИЧЕСКИЕ УРАВНЕНИЯ ЛИУВИЛЛЕВСКОГО ТИПА
}

\author{
А. В. ЖиБеР, В. В. Соколов
}

Статья является обзором результатов авторов, посвященных нелинейным гиперболическим уравнениям лиувиллевского типа. В качестве определения выбирается условие двустороннего обрыва цепочки инвариантов Лапласа для линеаризованного уравнения. В числе новых результатов приводится процедура нахождения общего решения и формулируется ответ в задаче классификации уравнений лиувиллевского типа.

Библиография: 46 названий.

\section{СОДЕРЖАНИЕ}

$\S 1$. Введение

$\S 2$. Преобразование и инварианты Лапласа ............................ 66

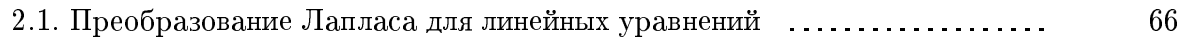

2.2. Инварианты Лапласа для линеаризованного уравнения … . . . . . . . . . 68

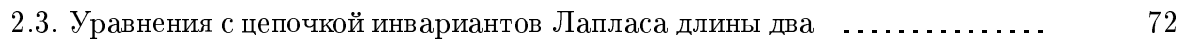

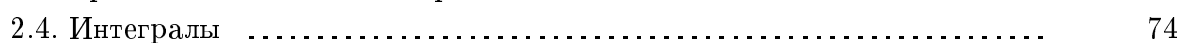

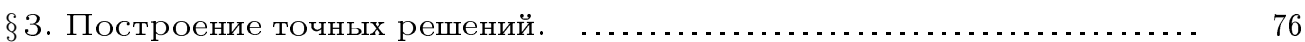

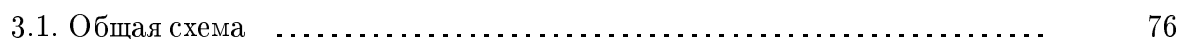

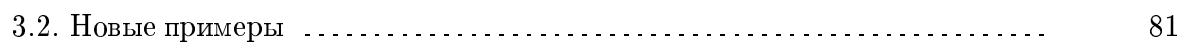

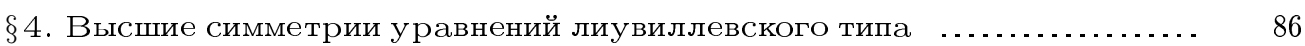

§5. Уравнения лиувиллевского типа и преобразования Миуры для эво-

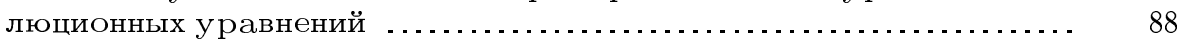

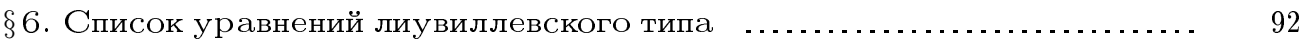

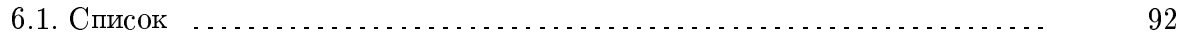

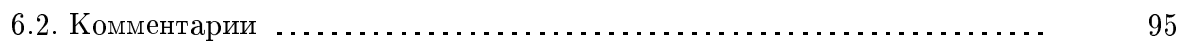

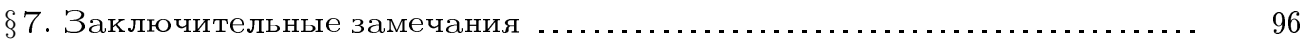

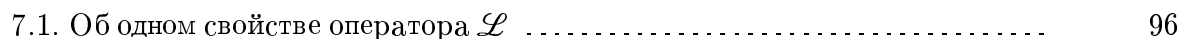

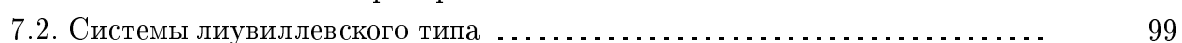

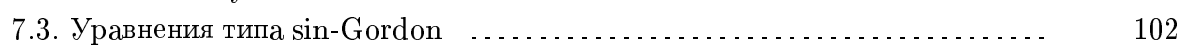

Список литературы . . . . . . . . . . . . . . . . . . . 104

Работа выполнена при финансовой поддержке Российского фонда фундаментальных исследований (гранты № № 99-01-00431 и 99-01-00294). Второй автор также был поддержан проектом INTAS (грант № 1782). 


\section{$\S$ 1. Введение}

На ранних этапах своего развития теория уравнений в частных производных в качестве одной из основных выдвигала проблему нахождения и исследования уравнений, допускающих явное интегрирование. Такие классики математики 18-19 веков, как Эйлер, Лагранж, Лиувилль, Лаплас, Дарбу, Ли, Якоби, Гурса и другие, разработали много приемов для нахождения точных решений дифференщиальных уравнений в частных производных (см. [1]-[7]).

В начале 20-го века сильнейшее влияние математической физики привело к переоценке ценностей в теории уравнений в частных производных. В результате ряд классических результатов, касаюшихся точного интегрирования, оказался основательно забыт даже специалистами. В настоящее время интерес к этим результатам значительно возрос в связи с открытием нового фундаментального метода точного интегрирования нелинейных уравнений в частных производных: метода обратной задачи рассеяния (см. [8], [9]).

Общеизвестньм примером точно интегрируемого нелинейного уравнения в частных производных является уравнение Лиувилля

$$
u_{x y}=\exp u .
$$

Изучение формальных свойств этого уравнения привело (см. [1], [6], [10]-[16]) к различным, вообще говоря неэквивалентным, определениям класса точно интегрируемых гиперболических уравнений лиувиллевского типа. В частности, в работах [17]-[20] в качестве определения было выбрано свойство конечности цепочки инвариантов Лапласа линеаризованного уравнения. В статье [21] этот подход был обобщен на случай произвольных уравнений второго порядка вида $F\left(x, y, u, u_{x}, u_{y}, u_{x x}, u_{x y}, u_{y y}\right)=0$.

Определение оказалось удачньм, поскольку позволило использовать несколько красивых классических тождеств (см. [3]), связанных с инвариантами Лапласа. В результате были найдены обшие формулы (см. §4), позволяюшие для таких уравнений в терминах инвариантов Лапласа описать высшие симметрии [19] и законы сохранения [17], [18]. Кроме того, была предложена единая процедура нахождения общего решения уравнения лиувиллевского типа. Соответствуюшие результаты впервые публикуются в $\S 3$ настоящей работы.

Для конкретного уравнения при проверке конечности цепочки используются только алгебраические операции и операция дифференцирования. Поэтому выкладки легко могут быть проделаны на компютере с помошью любой из систем аналитических вычислений.

Свойство обрьва цепочки инвариантов Лапласа может быть положено в основу классификации уравнений лиувиллевского типа. В результате попыток авторов полностью перечислить уравнения

$$
u_{x y}=F\left(x, y, u, u_{x}, u_{y}\right)
$$

лиувиллевского типа были найдены два новых примера таких уравнений. В $\S 3$ для этих примеров найдена явная формула для общего решения. 
Общий классификационный результат впервые сформулирован в $\S 6$ настоящей работы. Однако, поскольку вычисления оказались чрезвычайно трудоемкими и полное доказательство классификационной теоремы занимает около 200 страниц, имеется маленькая, но ненулевая вероятность ошибки в выкладках.

Отметим, что общая задача классификации интегрируемых уравнений (1.2), включающая в себя описание не только уравнений лиувиллевского типа, но и интегрируемых методом обратной задачи уравнений типа уравнения sin-Gordon $u_{x y}=\sin u$, является чрезвычайно сложной. Несмотря на частные результаты, полученные в рамках симметрийного подхода [22], [23], исследования далеки от своего завершения. Относительно недавно появился новьй пример такого уравнения [24], [25]. Это уравнение имеет вид

$$
u_{x y}=S(u) \sqrt{1-u_{x}^{2}} \sqrt{1-u_{y}^{2}} .
$$

Здесь $S$ - любое решение обыкновенного дифференциального уравнения вида

$$
S^{\prime \prime}-2 S^{3}+\lambda S=0
$$

где $\lambda$ - произвольная постоянная. В случае общего положения $S(u)$ - эллиптический синус Якоби. Уравнение (1.3) связано с уравнением

$$
v_{x y}=c \sin v
$$

дифференщиальной подстановкой

$$
v=\arcsin u_{x}+\arcsin u_{y}+P(u),
$$

где $P^{\prime 2}=2 S^{\prime}-2 S^{2}+\lambda$. Константа $c$ определяется значением первого интеграла $I=S^{\prime 2}-S^{4}+\lambda S^{2}$ с помошью формулы $I=c^{2}$.

Вырождение

$$
u_{x y}=\frac{1}{u} \sqrt{1-u_{x}^{2}} \sqrt{1-u_{y}^{2}}
$$

уравнения (1.3) является уравнением лиувиллевского типа и используется в качестве иллюстративного примера в $\S 3$.

В $\S 7$ настояшей работы содержится новое интегрируемое уравнение вида (1.2), связанное дифференциальной подстановкой с уравнением Цицейки. Там же приведен список известных в настоящий момент уравнений типа sin-Gordon и сформулирована гипотеза о его полноте.

В теории интегрируемых эволюционных уравнений хорошо известно преобразование Миуры $w=u_{x}-\frac{1}{2} u^{2}$, связываюшее модифицированное уравнение Кортевега-де Фриза

$$
u_{t}=u_{x x x}-\frac{3}{2} u^{2} u_{x}
$$

с уравнением Кортевега-де Фриза

$$
w_{t}=w_{x x x}+3 w w_{x} .
$$


Оказывается, что такие преобразования тесно связаны с гиперболическими уравнениями лиувиллевского типа. В частности, преобразование Миуры порождается уравнением $u_{x y}=u u_{y}$. Соответствуюшая теория изложена в $\S 5$.

$\mathrm{B} \S 7$ определение уравнений лиувиллевского типа обобщено на случай нелинейных гиперболических систем вида

$$
\vec{u}_{x y}=\vec{F}\left(x, y, \vec{u}, \vec{u}_{x}, \vec{u}_{y}\right) .
$$

Статья представляет собой обзор результатов авторов, полученных за последние шесть лет и частично опубликованных в [19], [20], [25].

Авторы благодарны Н. Камрану, М.В. Павлову, С. Я. Старцеву, Е. В. Ферапонтову, С.П. Цареву и А.Б. Шабату за внимание к работе и многочисленные полезные обсуждения.

\section{§. Преобразование и инварианты Лапласа}

2.1. Преобразование Лапласа для линейных уравнений. В классических работах (см. $[3],[26],[27])$ преобразования и инварианты Лапласа определялись для линейного гиперболического уравнения вида

$$
\left(\frac{\partial^{2}}{\partial x \partial y}+a(x, y) \frac{\partial}{\partial x}+b(x, y) \frac{\partial}{\partial y}+c(x, y)\right) V=0 .
$$

Нетрудно проверить, что функции

$$
h_{0}=\frac{\partial a}{\partial x}+a b-c, \quad k_{0}=\frac{\partial b}{\partial y}+a b-c
$$

являются инвариантами при преобразованиях вида $V \rightarrow \alpha(x, y) V$. Они называются главными инвариантами Лапласа уравнения (2.1).

Легко видеть, что уравнение (2.1) может быть переписано в виде системы

$$
\left(\frac{\partial}{\partial y}+a\right) V=V_{1}, \quad\left(\frac{\partial}{\partial x}+b\right) V_{1}=h_{0} V .
$$

Если $h_{0} \neq 0$, то функция $V_{1}$, определенная первой из формул (2.3), удовлетворяет уравнению

$$
\left(\frac{\partial^{2}}{\partial x \partial y}+a_{1}(x, y) \frac{\partial}{\partial x}+b_{1}(x, y) \frac{\partial}{\partial y}+c_{1}(x, y)\right) V_{1}=0,
$$

где

$$
a_{1}=a-\left(\ln h_{0}\right)_{y}, \quad b_{1}=b, \quad c_{1}=a_{1} b_{1}+b_{y}-h_{0} .
$$

Уравнение (2.4) называется $Y$-преобразованием Лапласа уравнения (2.1). Главные инварианты Лапласа уравнения (2.4) задаются формулами

$$
h_{1}=2 h_{0}-k_{0}-\left(\ln h_{0}\right)_{x y}, \quad k_{1}=h_{0} .
$$


Если $h_{1} \neq 0$, можно найти $Y$-преобразование Лапласа уравнения (2.4) и т. д. В результате получится цепочка уравнений

$$
\left(\frac{\partial^{2}}{\partial x \partial y}+a_{i}(x, y) \frac{\partial}{\partial x}+b_{i}(x, y) \frac{\partial}{\partial y}+c_{i}(x, y)\right) V_{i}=0, \quad i \in \mathbb{N},
$$

коэффициенты и инварианты которых связаны между собой соотношениями:

$$
\begin{gathered}
a_{i}=a_{i-1}-\left(\ln h_{i-1}\right)_{y}, \quad b_{i}=b_{i-1}, \quad c_{i}=a_{i} b_{i}+b_{y}-h_{i-1}, \\
h_{i}=2 h_{i-1}-h_{i-2}-\left(\ln h_{i-1}\right)_{x y}, \quad k_{i}=h_{i-1} .
\end{gathered}
$$

Здесь $a_{0}=a, b_{0}=b, c_{0}=c$.

Выясним, как связаны между собой решения уравнений (2.1) и (2.5). Запишем каждое из уравнений $(2.5)$ в виде системы

$$
\left(\frac{\partial}{\partial y}+a_{i}\right) V_{i}=V_{i+1}, \quad\left(\frac{\partial}{\partial x}+b\right) V_{i+1}=h_{i} V_{i}
$$

где

$$
a_{i}=a-\frac{\partial}{\partial y} \ln \left(h_{0} h_{1} \cdots h_{i-1}\right)
$$

Положим

$$
\psi=\exp \left(-\int a d y\right), \quad \bar{\psi}=\exp \left(-\int b d x\right) .
$$

Тогда формулы (2.7) могут быть переписаны в виде

$$
V_{1}=\psi \frac{\partial}{\partial y} \frac{1}{\psi}(V), \quad V_{i+1}=\psi h_{0} \cdot h_{i-1} \frac{\partial}{\partial y} \frac{1}{\psi h_{0} \cdot h_{i-1}}\left(V_{i}\right), \quad i \in \mathbb{N},
$$

и

$$
V_{i}=\frac{\bar{\psi}}{h_{i}} \frac{\partial}{\partial x} \frac{1}{\bar{\psi}}\left(V_{i+1}\right)
$$

Отсюда для любого $k \in \mathbb{N}$

$$
\begin{gathered}
V_{k+1}=\psi h_{0} \cdot h_{k-1} \frac{\partial}{\partial y} \frac{1}{h_{k-1}} \frac{\partial}{\partial y} \frac{1}{h_{k-2}} \cdots \frac{\partial}{\partial y} \frac{1}{h_{0}} \frac{\partial}{\partial y} \frac{1}{\psi}(V), \\
V=\frac{\bar{\psi}}{h_{0}} \frac{\partial}{\partial x} \frac{1}{h_{1}} \frac{\partial}{\partial x} \cdots \frac{1}{h_{k-2}} \frac{\partial}{\partial x} \frac{1}{h_{k-1}} \frac{\partial}{\partial x} \frac{1}{\bar{\psi}}\left(V_{k}\right) .
\end{gathered}
$$

Таким образом, если мы умеем решать уравнение (2.1), то можем решить и любое из уравнений (2.5), и наоборот.

$X$-преобразование Лапласа определяется аналогично. А именно, уравнение (2.1) эквивалентно системе

$$
\left(\frac{\partial}{\partial x}+b\right) V=V_{-1}, \quad\left(\frac{\partial}{\partial y}+a\right) V_{-1}=k_{0} V .
$$


Если для (2.1) инвариант $h_{-1} \stackrel{\text { def }}{=} k_{0}$ не равен нулю, то $V_{-1}$ удовлетворяет уравнению

$$
\left(\frac{\partial^{2}}{\partial x \partial y}+a_{-1}(x, y) \frac{\partial}{\partial x}+b_{-1}(x, y) \frac{\partial}{\partial y}+c_{-1}(x, y)\right) V_{-1}=0,
$$

которое называется $X$-преобразованием Лапласа уравнения (2.1). Продолжая этот процесс, получаем цепочку уравнений

$$
\left(\frac{\partial^{2}}{\partial x \partial y}+a_{-i}(x, y) \frac{\partial}{\partial x}+b_{-i}(x, y) \frac{\partial}{\partial y}+c_{-i}(x, y)\right) V_{-i}=0, \quad i \in \mathbb{N},
$$

коэффициенты и инварианты которых связаны между собой соотношениями:

$$
\begin{gathered}
a_{-i-1}=a_{-i}, \quad b_{-i-1}=b_{-i}-\left(\ln h_{-i-1}\right)_{x}, \\
c_{-i-1}=a_{-i-1} b_{-i-1}+a_{x}-h_{-i-1}, \\
h_{-i}=2 h_{-i-1}-h_{-i-2}-\left(\ln h_{-i-1}\right)_{x y}, \quad k_{-i}=h_{-i-1} .
\end{gathered}
$$

Формулы, аналогичные (2.10), (2.11), имеют вид

$$
\begin{aligned}
V_{-s-1} & =\bar{\psi} h_{-1} \cdot h_{-s} \frac{\partial}{\partial x} \frac{1}{h_{-s}} \frac{\partial}{\partial x} \frac{1}{h_{-s+1}} \cdots \frac{\partial}{\partial x} \frac{1}{h_{-1}} \frac{\partial}{\partial x} \frac{1}{\bar{\psi}}(V), \\
V & =\frac{\psi}{h_{-1}} \frac{\partial}{\partial y} \frac{1}{h_{-2}} \frac{\partial}{\partial y} \cdots \frac{1}{h_{-s+1}} \frac{\partial}{\partial y} \frac{1}{h_{-s}} \frac{\partial}{\partial y} \frac{1}{\psi}\left(V_{-s}\right) .
\end{aligned}
$$

ОПРЕДЕЛЕНИЕ 1. Множество главных инвариантов $h_{i}, i \in \mathbb{Z}$, уравнений $(2.5)$ и (2.14) назьвается последовательностью инвариантов Лапласа для уравнения (2.1).

Согласно формулам (2.6), (2.15) имеем $k_{i}=h_{i-1}, i \in \mathbb{Z}$, и поэтому все инварианты $k_{i}$ также содержатся в этой последовательности.

Последовательность инвариантов Лапласа однозначно определяется рекуррентной формулой

$$
h_{i}=2 h_{i-1}-h_{i-2}-\left(\ln h_{i-1}\right)_{x y}, \quad i \in \mathbb{Z},
$$

и начальными данными

$$
h_{0}=\frac{\partial a}{\partial x}+a b-c, \quad h_{-1}=\frac{\partial b}{\partial y}+a b-c .
$$

2.2. Инварианты Лапласа для линеаризованного уравнения. Для заданного решения $u_{0}(x, y)$ уравнения (1.2) формула $u=u_{0}+\varepsilon V+\cdots$ приводит к линейному уравнению для $V$, коэффициенты которого зависят от $u_{0}$. Поскольку это линеаризованное уравнение имеет вид (2.1), мы можем определить для него последовательность инвариантов Лапласа.

В дальнейшем мы намерены изучать свойства этих инвариантов, общие для всех решений уравнения (1.2). Для этого рассмотрим более формальную разновидность

$$
\left(D_{x} \circ D_{y}-\frac{\partial F}{\partial u_{x}} D_{x}-\frac{\partial F}{\partial u_{y}} D_{y}-\frac{\partial F}{\partial u}\right) V=0
$$


линеаризованного уравнения (1.2). Здесь $D_{x}=D$ и $D_{y}=\bar{D}$ - операторы полного дифференцирования по $x$ и $y$ в силу уравнения (1.2).

Операторы $D$ и $\bar{D}$ являются более сложными объектами, чем операторы частных производных. В частности, для некоторых уравнений (1.2) они имеют нетривиальные ядра, при этом не всегда верно, что решение уравнения $\bar{D} D(Z)=0$ имеет вид $Z=W+\bar{W}$, где $\bar{D}(W)=D(\bar{W})=0$, и т.д. Поэтому при работе с этими операторами требуется соблюдать определенную аккуратность и мы начнем со строгих определений.

Бо́льшая часть различных понятий в локальной теории нелинейных дифференциальных уравнений, таких как симметрия, первьй интеграл, закон сохранения, представление Лакса и т. д., определяется в терминах некоторых тождеств, которые должны быть вьполнены на любом решении $u$. Для этого необходимо исключать изо всех выражений те частные производные от $u$, которые можно выразить из уравнения и его дифференциальных следствий.

Например, когда речь идет о решениях уравнения $(1.2), u_{x y}$ всегда заменяется на правую часть $F\left(x, y, u, u_{x}, u_{y}\right), u_{x y y}$ - на выражение

$$
\frac{\partial F}{\partial y}+\frac{\partial F}{\partial u} u_{y}+\frac{\partial F}{\partial u_{x}} F+\frac{\partial F}{\partial u_{y}} u_{y y}
$$

и т. д. Легко видеть, что таким образом всякая смешанная производная от $u$ может быть выражена через

$$
\begin{gathered}
x, y, u, u_{1}=u_{x}, u_{2}=u_{x x}, u_{3}=u_{x x x}, \ldots, \\
\bar{u}_{1}=u_{y}, \bar{u}_{2}=u_{y y}, \bar{u}_{3}=u_{y y y}, \ldots
\end{gathered}
$$

Функции (2.19) нельзя связать между собой, пользуясь уравнением (1.2) и его дифференциальными следствиями. Поэтому во всех определениях и выкладках они считаются независимыми переменньми.

Операторы $D$ и $\bar{D}$ являются дифференцированиями в пространстве $\mathscr{F}$ локально аналитических функций, зависящих от конечного числа переменных (2.19). Эти дифференцирования однозначно задаются соотношениями

$$
\begin{gathered}
D\left(u_{k}\right)=u_{k+1}, \quad \bar{D}\left(\bar{u}_{k}\right)=\bar{u}_{k+1}, \quad u_{0}=\bar{u}_{0}=u, \quad k=0,1,2, \ldots \\
{[D, \bar{D}]=0, \quad D \bar{D}(u)=F\left(x, y, u, u_{1}, \bar{u}_{1}\right) .}
\end{gathered}
$$

Записанные как векторные поля, они имеют вид

$$
\begin{aligned}
& D=\frac{\partial}{\partial x}+\sum_{i=0}^{\infty} u_{i+1} \frac{\partial}{\partial u_{i}}+\sum_{i=1}^{\infty} \bar{D}^{i-1}(F) \frac{\partial}{\partial \bar{u}_{i}} \\
& \bar{D}=\frac{\partial}{\partial y}+\sum_{i=0}^{\infty} \bar{u}_{i+1} \frac{\partial}{\partial \bar{u}_{i}}+\sum_{i=1}^{\infty} D^{i-1}(F) \frac{\partial}{\partial u_{i}}
\end{aligned}
$$

Хотя с первого взгляда кажется, что в определении $D$ участвует $\bar{D}$ и наоборот, векторные поля корректно определены этими формулами. 
Поскольку определения преобразований и инвариантов Лапласа из раздела 2.1 являются чисто алгебраическими и используют только тот факт, что операторы частных производных являются коммутирующими дифференщированиями, они непосредственно обобщаются на случай линеаризованного уравнения (2.18). Для этого мы просто должны заменить во всех формулах операторы частных производных на полные производные $D$ и $\bar{D}$ в силу уравнения (1.2).

Согласно формулам (2.2) главные инварианты (2.18) имеют вид

$$
\begin{aligned}
& H_{1} \stackrel{\text { def }}{=} h_{0}=-D\left(\frac{\partial F}{\partial u_{1}}\right)+\frac{\partial F}{\partial u_{1}} \frac{\partial F}{\partial \bar{u}_{1}}+\frac{\partial F}{\partial u}, \\
& H_{0} \stackrel{\text { def }}{=} k_{0}=-\bar{D}\left(\frac{\partial F}{\partial \bar{u}_{1}}\right)+\frac{\partial F}{\partial u_{1}} \frac{\partial F}{\partial \bar{u}_{1}}+\frac{\partial F}{\partial u} .
\end{aligned}
$$

Отметим, что функции $H_{1}$ и $H_{0}$ остаются инвариантньми при преобразованиях вида $u \rightarrow \psi(x, y, u)$ в уравнении (1.2).

Остальные инварианты Лапласа рекуррентно определяются (ср. с (2.6), (2.15)) формулами

$$
D \bar{D}\left(\ln H_{i}\right)=-H_{i+1}-H_{i-1}+2 H_{i}, \quad i \in \mathbb{Z} .
$$

Легко видеть, что все инварианты являются рациональньми функциями от производных порядка выше чем 1. Например,

$$
\begin{gathered}
H_{1}=\alpha u_{2}+\beta, \quad \alpha=-\frac{\partial^{2} F}{\partial u_{1}^{2}}, \quad H_{0}=\overline{\alpha u}_{2}+\bar{\beta}, \quad \bar{\alpha}=-\frac{\partial^{2} F}{\partial \bar{u}_{1}^{2}}, \\
H_{2}=\frac{\bar{u}_{2}\left(p_{1} u_{3}+p_{2} u_{2}^{3}+p_{3} u_{2}^{2}+p_{4} u_{2}+p_{5}\right)+p_{6} u_{3}+p_{7} u_{2}^{3}+p_{8} u_{2}^{2}+p_{9} u_{2}+p_{10}}{\left(\alpha u_{2}+\beta\right)^{2}}
\end{gathered}
$$

где все коэффициенты $\alpha, \bar{\alpha}, \beta, \bar{\beta}$ и $p_{i}$ - некоторые конкретные функции от правой части $F$ уравнения (1.2) и ее частных производных по переменньм $x, y, u, u_{1}, \bar{u}_{1}$.

В частности,

$$
p_{2}=\frac{\partial^{3} F}{\partial u_{1}^{3}} \frac{\partial^{3} F}{\partial u_{1}^{2} \partial \bar{u}_{1}}-\frac{\partial^{2} F}{\partial u_{1}^{2}} \frac{\partial^{4} F}{\partial u_{1}^{3} \partial \bar{u}_{1}} .
$$

Структура инварианта $H_{-1}$ аналогична структуре $H_{2}$, но с заменой производных $u_{i}$ на $\bar{u}_{i}$ и наоборот.

Рост порядков производных в инвариантах Лапласа обусловлен тем, что оператор $\Delta=D \bar{D}$, фигурируюший в цепочке $(2.22)$, вообще говоря, повьшает порядок старших производных на единицу. Легко видеть, что при $i>0$ максимально возможный порядок $H_{i}$ по переменным $u_{2}, u_{3}, \ldots$ равен $i+1$, а по переменным $\bar{u}_{2}, \bar{u}_{3}, \ldots-$ равен $i$.

ОПРЕДЕЛЕНИЕ 2. Назовем (1.2) уравнением лиувиллевского типа, если существуют $r \geqslant 1$ и $s \geqslant 0$ такие, что $H_{r}=H_{-s} \equiv 0$.

В случае линейных уравнений (2.1) это определение приводит к детально изученному классиками классу уравнений с конечной цепочкой инвариантов Лапласа. Формулы для обшего решения таких уравнений и другую полезную информацию можно 
найти в [3]. В [28] было показано, что свойство конечности цепочки преобразований Лапласа эквивалентно факторизуемости сответствуюшего линейного оператора в некотором обобщенном смысле (разложимости на операторные идеалы "порядка 1").

Простейшим нелинейным уравнением лиувиллевского типа является уравнение (1.1). Для этого уравнения формулы (2.21) и (2.20) дают $H_{0}=H_{1}=\exp u$. Нетрудно проверить, что

$$
H_{2}=H_{-1}=0
$$

Другие примеры уравнений, удовлетворяюших условию (2.26), доставляют уравнения $4-7$ из $\S 6$.

Докажем следующее важное утверждение (ср. с [29], [23]).

Теорема 1. Для всякого уравнения лиувиллевского типа (1.2) существуют функиии $\psi\left(x, y, u, u_{1}, \ldots, u_{p}\right), \bar{\psi}\left(x, y, u, \bar{u}_{1}, \ldots, \bar{u}_{\bar{p}}\right)$ такие, что

$$
\frac{\partial F}{\partial u_{1}}=\bar{D} \ln \psi\left(x, y, u, u_{1}, \ldots, u_{p}\right), \quad \frac{\partial F}{\partial \bar{u}_{1}}=D \ln \bar{\psi}\left(x, y, u, \bar{u}_{1}, \ldots, \bar{u}_{\bar{p}}\right) .
$$

ЛЕмма 1. Пусть функиия $g\left(x, y, u, u_{1}, \ldots, u_{p}, \bar{u}_{1}, \ldots, \bar{u}_{q}\right)$, әде $p>0$, удовлетворяет соотношению

$$
D \bar{D}(g)=f\left(x, y, u, u_{1}, \ldots, u_{n}, \bar{u}_{1}, \ldots, \bar{u}_{m}\right), \quad n \leqslant p .
$$

Тогда

$$
\bar{D}\left(\frac{\partial g}{\partial u_{p}}\right)=-\frac{\partial F}{\partial u_{1}} \frac{\partial g}{\partial u_{p}}
$$

ДокАЗАТЕЛЬСТво. Легко проверить, что

$$
D \bar{D}(g)=u_{p+1}\left(\bar{D} \frac{\partial g}{\partial u_{p}}+\frac{\partial F}{\partial u_{1}} \frac{\partial g}{\partial u_{p}}\right)+\cdots .
$$

Здесь многоточием обозначены слагаемые, не зависяшие от $u_{p+1}$. Таким образом, если $p+1>n$, то вьполнено условие (2.26).

ДокАЗАТЕЛЬСТво тЕОРЕмЫ 1 . Если цепочка инвариантов $H_{i}$ обрывается, то существует инвариант $H_{n}$ максимального по переменньм $u_{i}$ порядка $k$. Если $k>0$, то, применяя лемму 1 к соотношению

$$
D \bar{D}\left(\ln H_{n}\right)=2 H_{n}-H_{n+1}-H_{n-1}
$$

и пользуясь формулой (2.29), приходим к первой из требуемых формул, где

$$
\psi=\left(\frac{\partial H_{n}}{\partial u_{k}}\right)^{-1}
$$

Если же все инварианты не зависят от $u_{1}, u_{2}, \ldots$, то из $(2.20)$ следует, что $\frac{\partial F}{\partial u_{1}}=0$ и можно положить $\psi=1$. Вторая из формул (2.27) вьводится аналогично.

Функции $\psi$ и $\bar{\psi}$ в дальнейшем играют ту же роль, что и функции (2.9) в формулах (2.10), (2.11). 
2.3. Уравнения с цепочкой инвариантов Лапласа длины два. Тождественное равенство нулю инварианта Лапласа эквивалентно тому, что функция $F$ удовлетворяет некоторой переопределенной системе уравнений в частных производных. Простейшие из них дают важную информацию об уравнениях с нулевьми инвариантами Лапласа.

Рассмотрим класс уравнений, для которых выполнено условие обрьва (2.26). Формула (2.25) показывает, что если $H_{2}=0$, то

$$
\frac{\partial^{2}}{\partial u_{1} \partial \bar{u}_{1}}\left(\ln \frac{\partial^{2} F}{\partial u_{1}^{2}}\right)=0
$$

Аналогично, из условия $H_{-1}=0$ следует, что

$$
\frac{\partial^{2}}{\partial u_{1} \partial \bar{u}_{1}}\left(\ln \frac{\partial^{2} F}{\partial \bar{u}_{1}^{2}}\right)=0
$$

Решая совместно уравнения (2.30) и (2.31), приходим к следующему утверждению.

ПРЕДЛОЖЕНИЕ 1. Если уравнение (1.2) удовлетворяет условиям (2.26) и

$$
\frac{\partial^{2} F}{\partial u_{1}^{2}} \frac{\partial^{2} F}{\partial \bar{u}_{1}^{2}} \neq 0
$$

то оно имеет следующую структуру:

$$
u_{x y}=P\left(x, y, u, u_{x}\right) \bar{P}\left(x, y, u, u_{y}\right)+Q_{1} u_{x} u_{y}+Q_{2} u_{x}+Q_{3} u_{y}+Q_{4},
$$

әде $Q_{i}$ - некоторье функции от переменных $x, y, u$.

ЗАмечАниЕ 1 . Легко видеть, что с помошью преобразования вида $u \rightarrow \psi(x, y, u)$ можно сделать функцию $Q_{1}$ нулем.

К сожалению, попытки дальнейшего прямолинейного использования условия (2.26) для уточнения вида функщий $P, \bar{P}_{\text {и }} Q_{i}$ наталкиваются на серьезные вычислительные трудности. Однако систематическое исследование системы

$$
D \bar{D} \ln H_{1}=2 H_{1}-H_{0}, \quad D \bar{D} \ln H_{0}=2 H_{0}-H_{1},
$$

эквивалентной условию (2.26), приводит к интересному результату. В этом разделе мы получим общую формулу для дифференциальной подстановки, связывающей удовлетворяюшие условиям предложения 1 уравнения (1.2) с волновьм уравнением $v_{x y}=0$.

Правая часть первого из соотношений (2.34) не зависит от третьих производных и является линейной функцией по вторым производным, в то время как $H_{1}$ задается формулой (2.23). 
ПРЕДЛОЖЕНИЕ 2. Пусть

$$
D \bar{D} \ln \left(a_{1} u_{2}+a_{2}\right)=b_{1} u_{2}+b_{2} \bar{u}_{2}+b_{3},
$$

где $a_{i}, b_{i}$ - некоторые функиии переменных $x, y, u, u_{1}, \bar{u}_{1}$. Тогда

$$
a_{1} u_{2}+a_{2}=q\left(x, y, u, u_{1}\right) \bar{q}\left(x, y, u, \bar{u}_{1}\right)\left(u_{2}+P\left(x, y, u, u_{1}\right)\right)
$$

для некоторых $q, \bar{q}$ и Р. При этом

$$
\begin{gathered}
\frac{\partial F}{\partial u_{1}}=\bar{D} \ln \left(u_{2}+P\right) \\
b_{1}=\frac{\partial^{2} F}{\partial u_{1}^{2}}+\left(\bar{D}+\frac{\partial F}{\partial u_{1}}\right)\left(\frac{\partial \ln q}{\partial u_{1}}\right), \quad b_{2}=\left(D+\frac{\partial F}{\partial \bar{u}_{1}}\right)\left(\frac{\partial \ln \bar{q}}{\partial \bar{u}_{1}}\right) .
\end{gathered}
$$

ДоКАЗАТЕЛЬСтво. Вообще говоря, левая часть (2.35) имеет вид (2.24). Приравнивая в этом выражении к нулю коэффициент $p_{1}$, находим, что $a_{2}=a_{1} P\left(x, y, u, u_{x}\right)$ для некоторого $P$. Равенство нулю коэффициента $p_{6}$ можно записать (это требует некоторой наблюдательности!) в виде (2.36). Наконец, условие $p_{2}=0$ эквивалентно тому, что

$$
a_{1} \frac{\partial^{2} a_{1}}{\partial u_{1} \partial \bar{u}_{1}}=\frac{\partial a_{1}}{\partial u_{1}} \frac{\partial a_{1}}{\partial \bar{u}_{1}},
$$

что дает $a_{1}=q\left(x, y, u, u_{1}\right) \bar{q}\left(x, y, u, \bar{u}_{1}\right)$. Формулы для коэффициентов $b_{1}$ и $b_{2}$ находятся прямой выкладкой.

Применяя предложение 2 к первому из соотношений (2.34), получаем, что

$$
-\frac{\partial^{2} F}{\partial u_{1}^{2}}=q\left(x, y, u, u_{1}\right) \bar{q}\left(x, y, u, \bar{u}_{1}\right) .
$$

Учитьвая "симметричное" соотношение

$$
-\frac{\partial^{2} F}{\partial \bar{u}_{1}^{2}}=r\left(x, y, u, u_{1}\right) \bar{r}\left(x, y, u, \bar{u}_{1}\right)
$$

приходим к формуле (2.33), где

$$
q=\frac{\partial^{2} P}{\partial u_{1}^{2}}, \quad \bar{q}=-\bar{P}, \quad \bar{r}=\frac{\partial^{2} \bar{P}}{\partial \bar{u}_{1}^{2}}, \quad r=-P .
$$

Соотношение (2.36) дает нам

$$
\frac{\partial F}{\partial u_{1}}=\bar{D}\left[\ln \left(H_{1}\right)-\ln \left(\frac{\partial^{2} F}{\partial u_{1}^{2}}\right)\right] .
$$

Это и симметричное к нему соотношение

$$
\frac{\partial F}{\partial \bar{u}_{1}}=D\left[\ln \left(H_{0}\right)-\ln \left(\frac{\partial^{2} F}{\partial \bar{u}_{1}^{2}}\right)\right]
$$

(cp. с (2.27)) чрезвычайно важны для полной классификации уравнений (1.2), (2.32) с обрывом (2.26). 
ТЕОРема 2. Всякое уравнение (1.2), удовлетворяющее условиям (2.32), (2.26), связано с волновым уравнением $v_{x y}=0$ дифференииальной подстановкой вида

$$
v=\Phi\left(x, y, u, u_{1}, \bar{u}_{1}, u_{2}, \bar{u}_{2}\right)
$$

әде

$$
\Phi=\ln \frac{H_{1}^{4}\left(\frac{\partial^{2} F}{\partial \bar{u}_{1}^{2}}\right)^{3}}{H_{0}^{4}\left(\frac{\partial^{2} F}{\partial u_{1}^{2}}\right)^{3}} .
$$

ДоКАЗАТЕльство. Вычитая из первого уравнения (2.34) второе, получим

$$
D \bar{D}\left(\ln \frac{H_{1}}{H_{0}}\right)=3\left(H_{1}-H_{0}\right)=3 \bar{D}\left(\frac{\partial F}{\partial \bar{u}_{1}}\right)-3 D\left(\frac{\partial F}{\partial u_{1}}\right) .
$$

Далее, воспользовавшись формулами (2.38) и (2.39), видим, что для функции $\Phi$, заданной формулой $(2.40)$, выполнено тождество $D \bar{D}(\Phi)=0$.

СлЕДСТВИЕ 1. Функции $\bar{W}=\bar{D}(\Phi)$ и $W=D(\Phi)$ удовлетворяют условиям $\bar{D}(W)=D(\bar{W})=0$.

2.4. Интегралы. Сушествование функций $W$ и $\bar{W}$ от переменных (2.19) таких, что $\bar{D}(W)=D(\bar{W})=0$ (см. следствие 1$)$, является одной из замечательных особенностей уравнений лиувиллевского типа.

ОПРЕДЕЛЕНИЕ 3. Функция $W\left(x, y, u, u_{1}, \ldots, u_{p}\right)$ называется $Y$-интегралом для уравнения $(1.2)$, если $\bar{D}(W)=0$. При этом предполагается, что $W$ зависит не только от переменной $x$.

Соотношение $\bar{D}(W)=0$ означает, что, подставив в $W$ любое решение $u(x, y)$ уравнения (1.2), мы получим некоторую (вообше говоря, разную для разных решений) функцию, зависящую только от $x$.

Аналогично, $X$-интеграл - это функция $\bar{W}\left(x, y, u, \bar{u}_{1}, \ldots, \bar{u} \bar{p}\right)$, удовлетворяющая соотношению $D(\bar{W})=0$.

Понятие $Y$-интеграла [16], [19], [20], [22], [23], [25], [30] в некотором смысле является обобщением понятия первого интеграла для обыкновенных дифференциальных уравнений. Однако здесь имеется сушественная разница. Всякое обькновенное уравнение обладает первыми интегралами, но, вообе говоря, найти нетривиальньй первьй интеграл ничуть не легче, чем решать само уравнение. Для уравнений в частных производных ситуация принципиально иная. А именно, лишш исключительные уравнения имеют $Y$-интегралы, но уж если известно, что у уравнения есть $Y$-интеграл данного порядка, то найти его не составляет особого труда.

Очевидно, что $Y$ - и $X$-интегралы могут быть определены как нетривиальные функции, зависяшие от переменных (2.19), которые постоянны вдоль характеристик. Поэтому по смыслу они очень близки к хорошо известньм в гидродинамике инвариантам Римана, которые, однако, зависят только от неизвестных функций (но не от их производных). 
Очевидно, что для любого $Y$-интеграла $w$ и функции $Q$ выражение

$$
W=Q\left(x, w, D(w), \ldots, D^{k}(w)\right)
$$

также является $Y$-интегралом. В работах [23], [29] доказано следующее обратное утверждение.

ПРЕДЛОЖЕНИЕ 3. Всякий $Y$-интеграл имеет вид (2.41), где w-некоторый $Y$-интеграл минимального порядка.

Интеграл $w$, фигурируюший в предложении 3 , мы будем называть минимальным. Легко видеть, что минимальньй $Y$-интеграл $w$ определен с точностью до замень $w \rightarrow$ $\varphi(x, w)$. Аналогичные утверждения, конечно, имеют место и для $X$-интегралов.

Для уравнения Лиувилля (1.1) минимальньми интегралами являются

$$
w=u_{2}-\frac{1}{2} u_{1}^{2}, \quad \bar{w}=\bar{u}_{2}-\frac{1}{2} \bar{u}_{1}^{2} .
$$

Сушествование интегралов может быть положено в основу определения уравнений лиувиллевского типа [2], [16], [30], [31]. Эквивалентность этого определения и определения 2 была доказана в работах [17]-[19]:

Теорема 3. Уравнение (1.2) обладает $Y$ - и X-интегралами

$$
W\left(x, y, u, u_{1}, \ldots, u_{p}\right), \quad \bar{W}\left(x, y, u, \bar{u}_{1}, \ldots, \bar{u}_{\bar{p}}\right)
$$

тогда и только тогда, когда $H_{r}=H_{-s} \equiv 0$ для некоторых $r$ us. При этом $r \leqslant p, s \leqslant \bar{p}-1$.

Во многих вопросах, связанных с уравнениями лиувиллевского типа (см. определение 2), чрезвычайно важной оказывается следуюшая

ТЕОРемА 4. Для любого уравнения (1.2) лиувиллевского типа коэффициенты линейного дифференииального оператора $\mathscr{L}$, заданного формулой

$$
\mathscr{L}=\frac{\bar{\psi}}{\psi} H_{0} H_{-1} \cdots H_{1-s} D \frac{1}{H_{1-s}} D \cdots \frac{1}{H_{0}} D \frac{1}{H_{1}} \cdots D \frac{1}{H_{r-1}} D \frac{\psi H_{1} \cdots H_{r-1}}{\bar{\psi}}
$$

являются $Y$-интегралами.

ДОКАЗАТЕЛЬСТВО. Поскольку $H_{r}=0$, формулы (2.7) при $i=r-1$ (с учетом сделанного сдвига индекса, из-за которого $\left.h_{i}=H_{i+1}\right)$ имеют вид

$$
\left(\bar{D}-\bar{D}\left(\ln \psi H_{1} \cdots H_{r-1}\right)\right) V_{r-1}=V_{r}, \quad\left(D-\frac{\partial F}{\partial \bar{u}_{1}}\right) V_{r}=0 .
$$

Положим $V_{r}=0$. Тогда $V_{r-1}=\psi H_{1} \cdots H_{r-1} Q(x)$ удовлетворяет $(2.44)$ для любой функции $Q(x)$. Формула $(2.11)$ дает

$$
V=\bar{\psi} \frac{1}{H_{1}} D \frac{1}{H_{2}} \cdots D \frac{1}{H_{r-1}} D\left(\frac{\psi}{\bar{\psi}} H_{1} \cdots H_{r-1} Q(x)\right)
$$


В соответствии с формулой (2.16)

$$
V_{-s-1}=\bar{\psi} H_{0} H_{-1} \cdots H_{1-s} D \frac{1}{H_{1-s}} D \cdots \frac{1}{H_{-1}} D \frac{1}{H_{0}} D \frac{1}{\bar{\psi}} V .
$$

Так как

$$
\left(\bar{D}-\frac{\partial F}{\partial u_{1}}\right) V_{-s-1}=0,
$$

имеем $\bar{D}\left(\psi^{-1} V_{-s-1}\right)=0$. Подставляя сюда полученные вьше формулы для $V_{-s-1}$ и $V$, получаем $\bar{D} \mathscr{L}(Q)=0$. В силу произвольности функции $Q(x)$ отсюда вытекает, что $\bar{D}(\mathscr{L})=0$. Теорема 4 доказана.

Аналогично доказывается, что коэффициенты оператора $\overline{\mathscr{L}}$ вида

$$
\overline{\mathscr{L}}=\frac{\psi}{\bar{\psi}} H_{1} \cdots H_{r-1} \bar{D} \frac{1}{H_{r-1}} \bar{D} \cdots \frac{1}{H_{0}} \bar{D} \frac{1}{H_{-1}} \bar{D} \cdots \frac{1}{H_{-s+1}} \bar{D} \frac{\bar{\psi} H_{0} \cdots H_{-s+1}}{\psi}
$$

являются $X$-интегралами.

ЗАмечАниЕ 2. Функция $\psi$ определена с точностью до умножения на $Y$-интегралы. Ее можно выбрать так, чтобы порядок $\psi$ был меньше порядка минимального $Y$-интеграла. Такая функция $\psi$ определена с точностью до умножения на функции от $x$. Из-за неоднозначности функции $\psi$ оператор $\mathscr{L}$ определен с точностью до преобразования $\mathscr{L} \rightarrow W \mathscr{L} W^{-1}$, где $W$ - произвольньй $Y$-интеграл.

Коэффициенты операторов $\mathscr{L}$ и $\overline{\mathscr{L}}$ абсолютно алгоритмически вычисляются с помощью компюютера, что дает самьй эффективный из известных авторам способов нахождения интегралов для заданного уравнения лиувиллевского типа.

\section{§ 3. Построение точных решений.}

3.1. Общая схема. Известная формула для обшего решения уравнения Лиувилля (1.1) имеет вид

$$
u(x, y)=\ln \left[\frac{2 Z^{\prime}(x) \bar{Z}^{\prime}(y)}{(Z(x)+\bar{Z}(y))^{2}}\right],
$$

где $Z(x)$ и $\bar{Z}(y)$ - произвольные функции. Подставляя это решение в (2.42), находим, что

$$
w=\frac{Z^{\prime \prime \prime}}{Z^{\prime}}-\frac{3}{2} \frac{Z^{\prime \prime 2}}{Z^{\prime 2}} .
$$

Важным для нас является тот факт, что функция $Z(x)$ параметризует ядро оператоpa $\mathscr{L}$. Действительно, согласно формуле (2.43)

$$
\mathscr{L}=\exp (u) D \exp (-u) D \exp (-u) D \exp (u)=D^{3}+\left(2 u_{x x}-u_{x}^{2}\right) D+u_{x x x}-u_{x} u_{x x} .
$$

Таким образом,

$$
\mathscr{L}=D^{3}+2 w D+w^{\prime}
$$


Хорошо известно, что базисом ядра этого оператора являются

$$
\varphi_{1}=\frac{1}{Z^{\prime}}, \quad \varphi_{2}=\frac{Z}{Z^{\prime}}, \quad \varphi_{3}=\frac{Z^{2}}{Z^{\prime}}
$$

где функция $Z$ связана с $w$ соотношением (3.2).

Покажем, что для любого уравнения лиувиллевского типа общее решение может быть записано в терминах ядер операторов $\mathscr{L}$ и $\overline{\mathscr{L}}$ и сопряженных к ним.

Рассмотрим функцию

$$
A=\frac{\bar{\psi}}{\psi H_{1} H_{2} \cdots H_{r-1}}
$$

Из формул $(2.43)$ и (2.46) следует, что $\mathscr{L}(A)=\overline{\mathscr{L}}^{*}(A)=0$. Здесь через $\overline{\mathscr{L}}^{*}$ обозначен оператор, формально сопряженньй к $\overline{\mathscr{L}}$. Таким образом,

$$
A=\sum_{i, j=1}^{s+r} c_{i j} \varphi_{i}(x) \bar{\varphi}_{j}(y) .
$$

Здесь $\varphi_{1}, \varphi_{2}, \ldots, \varphi_{s+r}-$ базис ядра $\mathscr{L}, \bar{\varphi}_{1}, \bar{\varphi}_{2}, \ldots, \bar{\varphi}_{s+r}-$ базис ядра $\overline{\mathscr{L}}^{*}$, а $c_{i j}-$ некоторые постоянные.

Аналогично, функция

$$
\bar{A}=\frac{\psi}{\bar{\psi} H_{0} H_{-1} \cdots H_{1-s}}
$$

удовлетворяет уравнениям $\overline{\mathscr{L}}(\bar{A})=\mathscr{L}^{*}(\bar{A})=0$.

Если мы знаем $A$ как функцию от $x$ и $y$, то с помощью алгебраических операций и взятия полных производных $D$ и $\bar{D}$ можно найти значения некоторых других выражений от переменных (2.19). Например, согласно следующей лемме все инварианты Лапласа для уравнения (1.2) выражаются через функцию (3.5).

Лемма 2. Справедливьц формульи

$$
H_{i}=D \bar{D} \mathscr{H}_{i}, \quad i=r-1, r-2, \ldots, 1-s,
$$

где величины $\mathscr{H}_{i}$ определяются рекуррентными соотношениями

$$
\begin{gathered}
\mathscr{H}_{i}=2 \mathscr{H}_{i+1}-\mathscr{H}_{i+2}-\ln D \bar{D} \mathscr{H}_{i+1}, \quad i=r-2, r-3, \ldots, 1-s, \\
\mathscr{H}_{r}=0, \quad \mathscr{H}_{r-1}=-\ln A .
\end{gathered}
$$

ДокАЗАТЕльСтво. Складывая соотношения цепочки

$$
H_{i+1}=2 H_{i}-H_{i-1}-D \bar{D} \ln H_{i}
$$

с номерами $i=r-1, i=r-2, \ldots, i=0$ и учитьвая, что $H_{r}=0$, получаем

$$
H_{r-1}+H_{1}-H_{0}-D \bar{D} \ln H_{1} \cdots H_{r-1}=0
$$


или

$$
H_{r-1}=D \bar{D} \ln H_{1} \cdots H_{r-1}+D F_{u_{1}}-\bar{D} F_{\bar{u}_{1}} .
$$

Так как согласно теореме $1 F_{u_{1}}=\bar{D} \ln \psi$ и $F_{\bar{u}_{1}}=D \ln \bar{\psi}$, то имеем

$$
H_{r-1}=D \bar{D} \ln \left(\frac{\psi}{\bar{\psi}} H_{1} \cdots H_{r-1}\right)=D \bar{D} \mathscr{H}_{r-1}
$$

где

$$
\mathscr{H}_{r-1}=-\ln A .
$$

Определим $\mathscr{H}_{r-2}$ формулой $(3.9): \mathscr{H}_{r-2}=2 \mathscr{H}_{r-1}-\ln D \bar{D} \mathscr{H}_{r-1}$, и применим к обеим частям этого равенства оператор $D \bar{D}$. Используя $(3.10)$, получим

$$
D \bar{D} \mathscr{H}_{r-2}=2 H_{r-1}-D \bar{D} \ln H_{r-1}=H_{r-2} \text {. }
$$

Аналогично доказьвается, что $D \bar{D} \mathscr{H}_{r-3}=H_{r-3}$ и т. д.

Отметим, что кроме рекуррентной формулы (3.9), существует следуюшее детерминантное представление для выражений $\mathscr{H}_{i}$ (см. [32]):

$$
\mathscr{H}_{r-k}=-\ln \left((-1)^{k(k+1) / 2} \Delta_{k+1}(A)\right), \quad k=1, \ldots, r+s-1,
$$

где $\Delta_{k}(A)$ - главньй минор размера $k$ матрицы $A_{i j}=D^{i-1} \bar{D}^{j-1} A$.

В практическом плане эти формулы можно использовать следующим образом. С одной стороны, мы знаем $A, \bar{A}, \bar{D}(A), D(\bar{A})$ и инварианты Лапласа как функции переменных (2.19). С другой стороны, в силу формул (3.6), (3.8) они известны нам как функции от $x$ и $y$. Поэтому с помошью теоремы о неявной функции можно найти $u$ вместе с $u_{1}, \bar{u}_{1}, \ldots$ как функции от $x$ и $y$. Это и даст нам формулу для общего решения $u(x, y)$. При этом в ходе вычислений находятся связи между константами $c_{i j}$ из формулы (3.6). Важно отметить, что эти константы, вообще говоря, не являются произвольными.

Конечно, в каждом конкретном случае эта схема допускает технические трюки, упрошаюшие вычисления. В частности, базис ядер операторов $\mathscr{L}, \overline{\mathscr{L}}, \mathscr{L}^{*}$ и $\overline{\mathscr{L}}^{*}$ гораздо проше описать, если эти операторы допускают факторизашию (см. примеры ниже). Кроме того, часто переменные $u, u_{1}, \bar{u}_{1}, \ldots$ удобнее находить не из тождеств для $A, \bar{A}, \bar{D}(A), D(\bar{A})$ и $H_{i}$, а используя другой, эквивалентньй, но более простой, набор тождеств.

ПРИмеР 1. В качестве простейшего примера покажем, как регулярным образом найти обшее решение (3.1) уравнения Лиувилля.

Как уже отмечалось, для уравнения Лиувилля

$$
\mathscr{L}=-\mathscr{L}^{*}=D^{3}+2 w D+w_{x}, \quad \overline{\mathscr{L}}=-\overline{\mathscr{L}}^{*}=\bar{D}^{3}+2 \bar{w} \bar{D}+\bar{w}_{y} .
$$

Обозначим через $Z(x)$ функцию, порождаюшую ядро оператора $\mathscr{L}$ с помошью формулы (3.4). Аналогично, базис ядра оператора $\overline{\mathscr{L}}^{*}$ образуют

$$
\bar{\varphi}_{1}=\frac{1}{\bar{Z}^{\prime}}, \quad \bar{\varphi}_{2}=\frac{\bar{Z}}{\bar{Z}^{\prime}}, \quad \bar{\varphi}_{3}=\frac{\bar{Z}^{2}}{\bar{Z}^{\prime}} .
$$


Согласно (3.5) $A=e^{-u}$, и формула (3.6) дает

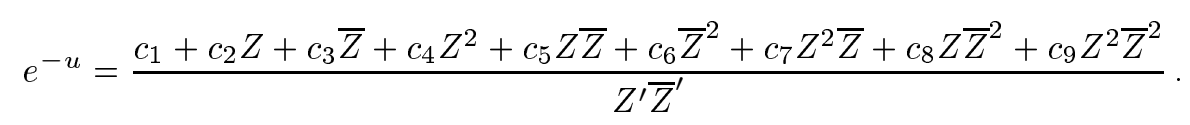

Для того чтобы определить постоянные $c_{i}$, заметим, что $\mathscr{H}_{0}=\mathscr{H}_{1}=u$ и, следовательно, соотношение (3.11) при $k=1$ имеет вид:

$$
A=\bar{D}(A) D(A)-A D \bar{D}(A)
$$

Подставляя в эту формулу явное выражение для $A$, получаем соотношения на коэффициенты $c_{i}$ :

$$
\begin{array}{lll}
c_{1}=c_{2} c_{3}-c_{1} c_{5}, & c_{2}=2 c_{3} c_{4}-2 c_{1} c_{7}, & c_{3}=2 c_{2} c_{6}-2 c_{1} c_{8}, \\
c_{4}=c_{4} c_{5}-c_{2} c_{7}, & c_{5}=4 c_{4} c_{6}-4 c_{1} c_{9}, & c_{6}=c_{5} c_{6}-c_{3} c_{8}, \\
c_{7}=2 c_{4} c_{8}-2 c_{9} c_{2}, & c_{8}=2 c_{7} c_{6}-2 c_{3} c_{9}, & c_{9}=c_{7} c_{8}-c_{5} c_{9} .
\end{array}
$$

Решение

$$
c_{1}=0, c_{2}=0, c_{3}=0, c_{4}=\frac{1}{2}, c_{5}=1, c_{6}=\frac{1}{2}, c_{7}=0, c_{8}=0, c_{9}=0
$$

этой системы соответствует (3.1). Нетрудно проверить, что все остальные решения с точностью до переопределения функций $Z$ и $\bar{Z}$ также приводят к формуле (3.1).

ПримеР 2. Рассмотрим в качестве второго примера уравнение (1.4). Для краткости положим $\beta=\sqrt{1-u_{x}^{2}}, \bar{\beta}=\sqrt{1-u_{y}^{2}}$. Для уравнения (1.4) минимальные $Y$ - и $X$-интегралы задаются формулами

$$
w=\frac{u_{2}}{\beta}-\frac{\beta}{u}, \quad \bar{w}=\frac{\bar{u}_{2}}{\bar{\beta}}-\frac{\bar{\beta}}{u},
$$

а в качестве функций $\psi$ и $\bar{\psi}$ (см. (2.27)) можно выбрать $\psi=\beta, \bar{\psi}=\bar{\beta}$. Легко проверить, что для этого уравнения, так же как и для уравнения Лиувилля, $H_{2}=H_{-1}=0$. Инварианты $H_{0}$ и $H_{1}$ имеют вид

$$
H_{0}=\frac{\bar{\beta} w}{u \beta^{2}}, \quad H_{-1}=\frac{\beta \bar{w}}{u \bar{\beta}^{2}}
$$

Используя формулы $(2.43),(2.46)$, прямым вычислением получаем, что

$$
\mathscr{L}=D\left(D^{2}+v_{1} D+v_{2}+e^{2 v}\right), \quad \overline{\mathscr{L}}=\bar{D}\left(\bar{D}^{2}+\bar{v}_{1} D+\bar{v}_{2}+e^{2 \bar{v}}\right)
$$

где $v=\ln w, \bar{v}=\ln \bar{w}$. Операторы, сопряженные к $\mathscr{L}, \overline{\mathscr{L}}$, имеют вид

$$
\mathscr{L}^{*}=-\left(D^{2}-v_{1} D+e^{2 v}\right) D, \quad \overline{\mathscr{L}}^{*}=-\left(\bar{D}^{2}-\bar{v}_{1} \bar{D}+e^{2 \bar{v}}\right) \bar{D} .
$$


Согласно формулам (3.5), (3.7)

$$
A=\frac{u \beta}{w}, \quad \bar{A}=\frac{u \bar{\beta}}{\bar{w}} .
$$

Таким образом, функции (3.13) являются решениями уравнений

$$
\mathscr{L}(A)=\overline{\mathscr{L}}^{*}(A)=\overline{\mathscr{L}}(\bar{A})=\mathscr{L}^{*}(\bar{A})=0 .
$$

Более того, нетрудно проверить, что

$$
\begin{array}{ll}
\left(D^{2}+v_{1} D+v_{2}+e^{2 v}\right) A=-1, & \left(\bar{D}^{2}-\bar{v}_{1} \bar{D}+e^{2 \bar{v}}\right) \bar{D} A=0, \\
\left(\bar{D}^{2}+\bar{v}_{1} \bar{D}+\bar{v}_{2}+e^{2 \bar{v}}\right) \bar{A}=-1, & \left(D^{2}-v_{1} D+e^{2 v}\right) D \bar{A}=0 .
\end{array}
$$

Положим

$$
w=-i \frac{X^{\prime}}{X}, \quad \bar{w}=-i \frac{Y^{\prime}}{Y},
$$

где $X(x)$ и $Y(y)$ - произвольные функции, $i^{2}=-1$. Тогда $X^{2} / X^{\prime}$ и $1 / X^{\prime}$ образуют базис ядра оператора $D^{2}+v_{1} D+v_{2}+e^{2 v}$. Базисом ядра оператора $\left(D^{2}-v_{1} D+e^{2 v}\right) D$ являются $1, P(x), Q(x)$, где

$$
P^{\prime}=X, \quad Q^{\prime}=\frac{1}{X} .
$$

Аналогично, $Y^{2} / Y^{\prime}$ и $1 / Y^{\prime}$ - базис ядра оператора $\bar{D}^{2}+\bar{v}_{1} \bar{D}+\bar{v}_{2}+e^{2 \bar{v}}$ и $1, \bar{P}(y), \bar{Q}(y)$, где

$$
\bar{P}^{\prime}=Y, \quad \bar{Q}^{\prime}=\frac{1}{Y},
$$

- базис ядра оператора $\left(\bar{D}^{2}-\bar{v}_{1} \bar{D}+e^{2 \bar{v}}\right) \bar{D}$.

Методом вариации постоянных из (3.15) нетрудно найти, что

$$
\begin{aligned}
& A=\left(c_{1} \frac{X^{2}}{X^{\prime}}+c_{2} \frac{1}{X^{\prime}}\right) \bar{P}+\left(c_{3} \frac{X^{2}}{X^{\prime}}+c_{4} \frac{1}{X^{\prime}}\right) \bar{Q}+\frac{1}{2} \frac{P}{X^{\prime}}-\frac{1}{2} \frac{X^{2}}{X^{\prime}} Q, \\
& \bar{A}=\left(\bar{c}_{1} \frac{Y^{2}}{Y^{\prime}}+\bar{c}_{2} \frac{1}{Y^{\prime}}\right) P+\left(\bar{c}_{3} \frac{Y^{2}}{Y^{\prime}}+\bar{c}_{4} \frac{1}{Y^{\prime}}\right) Q+\frac{1}{2} \frac{\bar{P}}{Y^{\prime}}-\frac{1}{2} \frac{Y^{2}}{Y^{\prime}} \bar{Q}
\end{aligned}
$$

где $c_{i}$ и $\bar{c}_{i}$ - некоторые постоянные.

Дифференщируя соотношения (3.13), приходим к равенствам

$$
\begin{array}{ll}
w \bar{D}(A)=u_{y} \beta-u_{x} \bar{\beta}, & \bar{w} D(\bar{A})=u_{x} \bar{\beta}-u_{y} \beta \\
D(w A)=-u u_{x} w, & \bar{D}(\bar{w} \bar{A})=-u u_{y} \bar{w} .
\end{array}
$$

Левые части этих равенств как функции от $x$ и $y$ нам известны. Выразим из этих соотношений $u_{x}, u_{y}$ и $u$ через произвольные функции $X(x)$ и $Y(y)$.

Из (3.20) следует, что $w \bar{D}(A)=-\bar{w} D(\bar{A})$. Подставляя сюда выражения (3.16) и (3.19), находим, что

$$
\bar{c}_{1}=-c_{1}, \quad \bar{c}_{2}=-c_{3}, \quad \bar{c}_{3}=-c_{2}, \quad \bar{c}_{4}=-c_{4} .
$$


Согласно формулам (3.13) и (3.21)

$$
\beta=\frac{A w}{u}, \quad \bar{\beta}=\frac{\bar{A} \bar{w}}{u}, \quad u_{x}=-\frac{D(w A)}{u w}, \quad u_{y}=-\frac{\bar{D}(\bar{w} \bar{A})}{u \bar{w}} .
$$

Поскольку $\beta^{2}=1-u_{x}^{2}$ и $\bar{\beta}^{2}=1-u_{y}^{2}$, отсюда получаем

$$
u^{2}=A^{2} w^{2}-\left(\frac{D(A w)}{w}\right)^{2}=\bar{A}^{2} \bar{w}^{2}-\left(\frac{\bar{D}(\bar{A} \bar{w})}{\bar{w}}\right)^{2} .
$$

Из второго равенства в этой цепочке следует, что

$$
c_{1}=c_{4}=0, \quad 4 c_{2} c_{3}+1=0 .
$$

Поскольку функции $X(x)$ и $Y(y)$ определены с точностью до пропорциональности, без ограничения общности можно положить $c_{2}=\frac{1}{2}, c_{3}=-\frac{1}{2}$. Теперь первое равенство дает следуюший окончательньй ответ:

$$
u(x, y)=\sqrt{(Q(x)+\bar{Q}(y))(P(x)+\bar{P}(y))},
$$

где

$$
P^{\prime} Q^{\prime}=\bar{P}^{\prime} \bar{Q}^{\prime}=1
$$

3.2. Новые примеры. Почти все примеры уравнений лиувиллевского типа содержатся в классических работах (см. [3]). В частности, хорошо известно уравнение (1.4), рассмотренное вьше.

В результате попыток авторов полностью перечислить все уравнения лиувиллевского типа были найдены два новых примера таких уравнений. Первое из найденных уравнений имеет вид

$$
u_{x y}=\frac{1}{6 u+y} B^{2}(B-1) \bar{B}(\bar{B}-1)^{2}+\frac{1}{6 u+x} \bar{B}^{2}(\bar{B}-1) B(B-1)^{2},
$$

где $B=B\left(u_{x}\right)$ и $\bar{B}=\bar{B}\left(u_{y}\right)$ - решения кубических уравнений

$$
\frac{1}{3} B^{3}-\frac{1}{2} B^{2}=u_{x}, \quad \frac{1}{3} \bar{B}^{3}-\frac{1}{2} \bar{B}^{2}=u_{y} .
$$

Правая часть второго из этих уравнений

$$
u_{x y}=\frac{1}{u} b\left(u_{x}\right) \bar{b}\left(u_{y}\right)
$$

также содержит кубические радикалы от первых производных. А именно, функции $b\left(u_{x}\right)$ и $\bar{b}\left(u_{y}\right)$ определяются как решения следуюших кубических уравнений

$$
\left(u_{x}-b\right)\left(b+2 u_{x}\right)^{2}=1, \quad\left(u_{y}-\bar{b}\right)\left(\bar{b}+2 u_{y}\right)^{2}=1 .
$$

В статье [25] приведена формула для общего решения уравнения (3.22). Эта формула была получена с помошњю обсуждаемого в настоящей работе метода. Ниже мы показываем, как он работает, на примере уравнений (3.22), (3.24).

Вычисления, связанные с этими уравнениями, довольно громоздки. Заметно упрощает ситуащию тот факт, что в обоих случаях оператор $\mathscr{L}$ удается разложить в произведение двух операторов, коэффициенты которых по-прежнему являются $Y$-интегралами. По-видимому, это связано с существованием дифференциальных подстановок, сводяших наши уравнения к более простым. Явные формулы для этих подстановок приведены ниже. 
ПримеР 3. Рассмотрим уравнение (3.24). Нетрудно проверить, что для этого уравнения $H_{3}=H_{-2}=0$. Функция

$$
w=\frac{1}{2}\left(\frac{p^{2}}{2}+\frac{3}{2} \frac{b p}{u}+3 \frac{u_{1} p}{u}+D p\right)
$$

где

$$
p=2 \frac{u_{2}}{b}+\frac{b}{u},
$$

является минимальным $Y$-интегралом для (3.24). При таком выборе функции $w$ (которая определена с точностью до замены $w \rightarrow \varphi(x, w))$ коэффициенты оператора $\mathscr{L}$, заданного формулой (2.43), выглядят особенно просто. Оказьвается, что $\mathscr{L}$ раскладывается в произведение $\mathscr{L}=\mathscr{L}_{1} \mathscr{L}_{2}$, где

$$
\mathscr{L}_{1}=D^{3}-4 w D-2 D(w), \quad \mathscr{L}_{2}=D^{2}+D(\ln w) D+D^{2}(\ln w)-w
$$

Отметим, что

$$
\mathscr{L}_{1}^{*}=-\mathscr{L}_{1}, \quad \mathscr{L}_{2}^{*}=D^{2}-D(\ln w) D-w=w \mathscr{L}_{2} \frac{1}{w}
$$

Аналогичные формулы имеют место и для оператора $\overline{\mathscr{L}}$.

Поскольку для уравнения (3.24) $\psi=b, \bar{\psi}=\bar{b}$, основные соотношения (3.14) имеют вид

$$
\begin{aligned}
\mathscr{L}\left(\frac{\bar{b}}{b H_{1} H_{2}}\right) & =0, & \mathscr{L}^{*}\left(\frac{b}{\bar{b} H_{0} H_{-1}}\right) & =0, \\
\overline{\mathscr{L}}\left(\frac{b}{\bar{b} H_{0} H_{-1}}\right) & =0, & \overline{\mathscr{L}}^{*}\left(\frac{\bar{b}}{b H_{1} H_{2}}\right) & =0 .
\end{aligned}
$$

Нетрудно проверить, что функция $A$, определенная формулой (3.5), имеет вид

$$
A=\frac{u^{3} p\left(b+2 u_{1}\right)}{\left(\bar{u}_{1}-\bar{b}\right) w}
$$

Обозначим $\Phi=A w$, т.е.

$$
\Phi=\frac{u^{3} p\left(b+2 u_{1}\right)}{\bar{u}_{1}-\bar{b}} .
$$

Кроме того, полезно ввести обозначения

$$
F=\frac{u^{2}}{\left(\bar{u}_{1}-\bar{b}\right)\left(u_{1}-b\right)}
$$

и

$$
G=\sqrt{F}\left[\frac{3}{2} D \ln F \cdot \bar{D} \ln F-\frac{6}{F}\right]
$$

Аналогичньми формулами определяются $\bar{w}, \bar{p}, \overline{\mathscr{L}}, \overline{\mathscr{L}}_{1}, \overline{\mathscr{L}}_{2}, \bar{A}$ и $\bar{\Phi}$. 
Можно проверить, что соотношения (3.29) эквивалентны системе

$$
\begin{gathered}
\mathscr{L}_{1}(F)=\overline{\mathscr{L}}_{1}(F)=0, \quad \mathscr{L}_{2}\left(\frac{\Phi}{w}\right)=\overline{\mathscr{L}}_{2}\left(\frac{\bar{\Phi}}{\bar{w}}\right)=3 F, \\
\mathscr{L}_{1}(\bar{\Phi})=\overline{\mathscr{L}}_{1}(\Phi)=G, \quad \mathscr{L}_{2}^{*}(G)=\overline{\mathscr{L}}_{2}^{*}(G)=0 .
\end{gathered}
$$

В соответствии с общей схемой мы должны параметризовать двумя произвольньми функциями ядра всех встречаюшихся в (3.34) операторов, решить эту систему и найти явное выражение для функций $A=\Phi / w$ и $\bar{A}=\bar{\Phi} / \bar{w}$.

Перед тем как заняться этой технической вькладкой, покажем, что обшее решение уравнения (3.24) выражается через $F, \Phi$ и $\bar{\Phi}$.

Дифференцированием из (3.32) получаем, что

$$
p=D \ln F-\frac{2 u_{1}+b}{u}, \quad \bar{p}=\bar{D} \ln F-\frac{2 \bar{u}_{1}+\bar{b}}{u} .
$$

Используя эти соотношения и формулы (3.25), (3.31), находим

$$
u\left(b+2 u_{1}\right)\left(u_{1}-b\right)=\frac{\Phi+F}{D F} .
$$

Аналогично,

$$
u\left(\bar{b}+2 \bar{u}_{1}\right)\left(\bar{u}_{1}-\bar{b}\right)=\frac{\bar{\Phi}+F}{\bar{D} F} .
$$

Возведя в квадрат и перемножив эти тождества, в силу (3.25) получаем

$$
u^{4}\left(u_{1}-b\right)\left(\bar{u}_{1}-\bar{b}\right)=\frac{(\Phi+F)^{2}(\bar{\Phi}+F)^{2}}{(D F)^{2}(\bar{D} F)^{2}},
$$

откуда согласно (3.32)

$$
u^{6}=\frac{F(\Phi+F)^{2}(\bar{\Phi}+F)^{2}}{(D F)^{2}(\bar{D} F)^{2}} .
$$

Таким образом, общее решение исходного уравнения (3.24) допускает следуюшее представление:

$$
u=F^{1 / 6}\left[\frac{(\Phi+F)(\bar{\Phi}+F)}{D F \cdot \bar{D} F}\right]^{1 / 3} .
$$

Вернемся к исследованию системы (3.34). Нетрудно проверить, что функция $v=$ $-\ln (F / 2)$ является решением уравнения Лиувилля $v_{x y}=\exp (v)$. Другими словами, уравнение (3.24) связано с уравнением Лиувилля дифференциальной подстановкой

$$
v=-\ln \left[\frac{u^{2}}{2\left(\bar{u}_{1}-\bar{b}\right)\left(u_{1}-b\right)}\right] .
$$

Поэтому согласно (3.1) функция $F$ имеет вид

$$
F=\frac{(Z(x)+\bar{Z}(y))^{2}}{Z^{\prime}(x) \bar{Z}^{\prime}(y)} .
$$


Удобно выбрать $Z$ и $\bar{Z}$ в качестве произвольных параметризуюших функций.

Легко проверить, что

$$
G=-\frac{3}{2}\left(X_{1} Y_{2}+X_{2} Y_{1}\right)
$$

где

$$
\begin{array}{lr}
X_{1}(x)=\frac{2 Z^{\prime 2}-Z Z^{\prime \prime}}{Z^{\prime 3 / 2}}, & X_{2}(x)=\frac{Z^{\prime \prime}}{Z^{\prime 3 / 2}}, \\
Y_{1}(y)=\frac{2 \bar{Z}^{\prime 2}-\overline{Z Z^{\prime \prime}}}{\bar{Z}^{\prime 3 / 2}}, & Y_{2}(y)=\frac{\bar{Z}^{\prime \prime}}{\bar{Z}^{\prime 3 / 2}} .
\end{array}
$$

Функции $X_{1}$ и $X_{2}$ образуют базис ядра оператора $\mathscr{L}_{2}^{*}$. Согласно (3.28) базисом ядра для $\mathscr{L}_{2}$ служат $\frac{X_{1}}{w}$ и $\frac{X_{2}}{w}$. Наконец, как уже упоминалось в предыдущем разделе, базис ядра $\mathscr{L}_{1}$ задается формулой (3.4). Аналогичные формулы имеют место и для ядер операторов $\overline{\mathscr{L}}_{1}, \mathscr{L}_{2}$ и $\mathscr{L}_{2}^{*}$.

Теперь нетрудно выразить функции $\Phi$ и $\bar{\Phi}$ через $Z(x)$ и $\bar{Z}(y)$. Пусть функции $P_{1}$, $P_{2}, P_{3}, \bar{Q}_{1}, \bar{Q}_{2}$ - произвольные решения линейных уравнений:

$$
\begin{gathered}
\mathscr{L}_{2}\left(P_{1}\right)=\frac{3 Z^{2}}{Z^{\prime}}, \quad \mathscr{L}_{2}\left(P_{2}\right)=\frac{6 Z}{Z^{\prime}}, \quad \mathscr{L}_{2}\left(P_{3}\right)=\frac{3}{Z^{\prime}}, \\
\mathscr{L}_{1}\left(\bar{Q}_{1}\right)=-\frac{3}{2} X_{1}, \quad \mathscr{L}_{1}\left(\bar{Q}_{2}\right)=-\frac{3}{2} X_{2} .
\end{gathered}
$$

Аналогично определим $\bar{P}_{1}, \bar{P}_{2}, \bar{P}_{3}, Q_{1}, Q_{2}$.

Используя приведенные вьше формулы для $F$ и $G$, находим из (3.34), что

$$
\begin{aligned}
& \Phi=\frac{w}{\bar{Z}^{\prime}}\left(P_{1}(x)+P_{2}(x) \bar{Z}+P_{3}(x) \bar{Z}^{2}\right)+X_{2} Q_{1}(y)+X_{1} Q_{2}(y) \\
& \bar{\Phi}=\frac{\bar{w}}{Z^{\prime}}\left(\bar{P}_{1}(y)+\bar{P}_{2}(y) Z+\bar{P}_{3}(y) Z^{2}\right)+Y_{2} \bar{Q}_{1}(x)+Y_{1} \bar{Q}_{2}(x) .
\end{aligned}
$$

У системы (3.38) удается найти довольно простое частное решение, задаваемое формулами

$$
\begin{gathered}
\bar{Q}_{1}=-Z \bar{Q}_{2}, \quad \bar{Q}_{2}=-\left(\frac{w P_{1}}{X_{1} Z^{\prime}}+\frac{w Z P_{3}}{X_{2} Z^{\prime}}+\frac{Z}{X_{2} Z^{\prime 2}}+\frac{Z^{2}}{X_{1} Z^{\prime 2}}\right), \\
P_{1}=\left(C_{1}(x)-\frac{Z^{2}}{X_{1} Z^{\prime}}\right) \frac{X_{1}}{w}, \quad P_{2}=-\left(\frac{4}{X_{1} X_{2} w}+\frac{X_{2} P_{1}}{X_{1}}+\frac{X_{1} P_{3}}{X_{2}}\right), \\
P_{3}=-\left(C_{2}(x)+\frac{1}{X_{2} Z^{\prime}}\right) \frac{X_{2}}{w},
\end{gathered}
$$

где

$$
C_{1}^{\prime}(x)=\frac{Z}{\sqrt{Z^{\prime}}}, \quad C_{2}^{\prime}(x)=\frac{1}{\sqrt{Z^{\prime}}} .
$$

Десять произвольных постоянных, которые имеются в общем решении системы (3.38), уточняются весьма трудоемкой прямой подстановкой формулы (3.35) в 
уравнение (3.24). Оказьвается, что без ограничения общности все их можно положить нулями. Окончательньй ответ выглядит следуюшим образом:

$$
u(x, y)=\left[\frac{\left(\bar{C}_{1}-C_{1}+\bar{C}_{2} Z+C_{2} Z\right)\left(\bar{C}_{1}-C_{1}-\bar{C}_{2} \bar{Z}-C_{2} \bar{Z}\right)}{Z+\bar{Z}}\right]^{1 / 3} .
$$

Отметим, что в ответе все-таки имеются четыре произвольные постоянные, содержашиеся неявно в (3.40) и в формуле

$$
\bar{C}_{1}^{\prime}(y)=\frac{\bar{Z}}{\sqrt{\bar{Z}^{\prime}}}, \quad \bar{C}_{2}^{\prime}(y)=\frac{1}{\sqrt{\bar{Z}^{\prime}}} .
$$

ПримеР 4. Приведем ключевые формулы для уравнения (3.22). Для этого уравнения, как и для уравнения (3.24), $H_{3}=H_{-2}=0$. Явный вид минимальных $Y$ и $X$-интегралов $w$ и $\bar{w}$ можно найти в $\S 6$. Функции $\psi$ и $\bar{\psi}($ см. $(2.27))$ определяются формулами

$$
\begin{aligned}
& \psi=u_{2}-\frac{B^{4}(B-1)^{2}}{6 u+y}-\frac{B^{2}(B-1)^{4}}{6 u+x} \\
& \bar{\psi}=\bar{u}_{2}-\frac{\bar{B}^{4}(\bar{B}-1)^{2}}{6 u+x}-\frac{\bar{B}^{2}(\bar{B}-1)^{4}}{6 u+y} .
\end{aligned}
$$

Инварианты Лапласа уравнения (3.22) имеют вид

$$
\begin{aligned}
H_{1} & =\frac{\bar{B}(\bar{B}-1)}{B(B-1)}\left[\frac{\bar{B}-1}{(6 u+y)(B-1)^{2}}-\frac{\bar{B}}{(6 u+x) B^{2}}\right] \psi, \\
H_{0} & =\frac{B(B-1)}{\bar{B}(\bar{B}-1)}\left[\frac{B-1}{(6 u+x)(\bar{B}-1)^{2}}-\frac{B}{(6 u+y) \bar{B}^{2}}\right] \bar{\psi}, \\
H_{2} & =\frac{2(6 u+y)(6 u+x)}{\bar{B}(\bar{B}-1)\left[(6 u+x) B^{2}(\bar{B}-1)-(6 u+y) \bar{B}(B-1)^{2}\right]^{2}} \psi \bar{\psi}, \\
H_{-1} & =\frac{2(6 u+y)(6 u+x)}{B(B-1)\left[(6 u+y) \bar{B}^{2}(B-1)-(6 u+x) B(\bar{B}-1)^{2}\right]^{2}} \psi \bar{\psi}
\end{aligned}
$$

Операторы $\mathscr{L}$ и $\overline{\mathscr{L}}$ факторизуются:

$$
\begin{aligned}
& \mathscr{L}=D(D+w)(D+w)(D+2 w)(D+3 w), \\
& \overline{\mathscr{L}}=\bar{D}(\bar{D}+\bar{w})(\bar{D}+\bar{w})(\bar{D}+2 \bar{w})(\bar{D}+3 \bar{w}) .
\end{aligned}
$$

Функции $A$ и $\bar{A}$, удовлетворяющие системе (3.14), имеют вид

$$
\begin{aligned}
& A=\frac{1}{2 \psi^{3}}\left[(6 u+x)(\bar{B}-1) B^{5}(B-1)^{3}-(6 u+y) \bar{B} B^{3}(B-1)^{5}\right] \\
& \bar{A}=\frac{1}{2 \bar{\psi}^{3}}\left[(6 u+y)(B-1) \bar{B}^{5}(\bar{B}-1)^{3}-(6 u+x) B \bar{B}^{3}(\bar{B}-1)^{5}\right] .
\end{aligned}
$$


Минимальные интегралы $w$ и $\bar{w}$ удобно параметризовать произвольными функциями $X(x)$ и $Y(y)$ следуюшим образом:

$$
w=\frac{X^{\prime \prime \prime}(x)}{X^{\prime \prime}(x)}, \quad \bar{w}=\frac{Y^{\prime \prime \prime}(y)}{Y^{\prime \prime}(y)} .
$$

Не приводя технических подробностей, отметим только, что, в сравнении с выкладками из примера 3 , процедура нахождения функций $A$ и $\bar{A}$ из системы (3.14) заметно проше, поскольку все факторы в формулах $(3.41)$ имеют первый порядок и нет никаких проблем с описанием ядер операторов $\mathscr{L}, \overline{\mathscr{L}}, \mathscr{L}^{*}$ и $\overline{\mathscr{L}}^{*}$.

В процессе вычислений возникают функции $P(x), Q(x), \bar{P}(y)$ и $\bar{Q}(y)$, связанные с $X(x)$ и $Y(y)$ дифференциальными соотношениями:

$$
P^{\prime}=X^{\prime 2}, \quad \bar{P}^{\prime}=Y^{\prime 2}, \quad Q^{\prime}=X^{\prime 3}, \quad \bar{Q}^{\prime}=Y^{\prime 3} .
$$

В эти формулы в статье [25] вкралась опечатка.

Окончательный ответ вьглядит следуюшим образом:

$$
u(x, y)=-\frac{x+y}{12}+\frac{K_{1}}{12 \sqrt{K_{2}}},
$$

где

$$
\begin{aligned}
K_{1}= & 2(X+Y)^{3}+3(x-y)(X+Y)(\bar{P}-P)+(x-y)^{2}(\bar{Q}+Q), \\
K_{2}= & (x-y)^{2}(\bar{Q}+Q)^{2}-2(x-y)(\bar{P}-P)\left[2(\bar{P}-P)^{2}-3(\bar{Q}+Q)(X+Y)\right] \\
& +4(X+Y)^{3}(\bar{Q}+Q)-3(X+Y)^{2}(\bar{P}-P) .
\end{aligned}
$$

Отметим, что уравнение (3.22) связано дифференциальной подстановкой

$$
v=-\ln \left[\frac{A(A-1)(1-A-B)}{\psi}\right]
$$

с хорошо известньм уравнением лиувиллевского типа

$$
v_{x y}=\exp (v) v_{y} .
$$

\section{§4. Высшие симметрии уравнений лиувиллевского типа}

ОПРЕДЕЛЕнИЕ 4. Инфинитезимальной (локальной) симметрией уравнения (1.2) называется всякая функция $V\left(x, y, u, u_{x}, \ldots, u_{n}, u_{y}, \ldots, \bar{u}_{m}\right)$ от переменных $(2.19)$, удовлетворяюшая линеаризованному уравнению (2.18).

Это определение эквивалентно условию, что поток в силу эволюционного уравнения

$$
u_{\tau}=V\left(x, y, u, u_{x}, \ldots, u_{n}, u_{y}, \ldots, \bar{u}_{m}\right)
$$


переводит решения уравнения (1.2) снова в решения. Часто симметрией назьвают само эволюционное уравнение (4.1).

Если $n \leqslant 1, m \leqslant 1$, симметрия назьвается классической. В этом случае уравнение (4.1) может быть проинтегрировано методом характеристик, и ответ задает однопараметрическую групш точечных или контактных преобразований, действующую на множестве всех решений уравнения (1.2). Переменная $\tau$ играет роль группового параметра.

В противном случае симметрия называется высшей.

Хорошо известно, что сушествует два типа интегрируемых нелинейных уравнений (1.2). К первому относятся уравнения, своими свойствами похожие на уравнение Лиувилля. Они могут быть проинтегрированы в квадратурах. Наиболее известным из уравнений второго типа является уравнение $\sin -$ Gordon $u_{x y}=\sin u$. Эти уравнения интегрируются методом обратной задачи рассеяния.

Как те, так и другие уравнения имеют высшие симметрии. Поэтому проблема перечисления всех интегрируемых случаев строго может быть сформулирована как задача классификации уравнений (1.2), обладаюших высшими симметриями [33]-[36]. Последняя задача является весьма сложной и в настояшее время решена только для нескольких частных случаев (см. [29]). Например, в [22] доказано, что нелинейное уравнение Клейна-Гордона

$$
u_{x y}=F(u)
$$

обладает высшими симметриями тогда и только тогда, когда оно эквивалентно либо уравнению Лиувилля, либо уравнению sin-Gordon, либо уравнению Цицейки

$$
u_{x y}=\exp (u)+\exp (-2 u)
$$

В работе [22] показано, что все высшие симметрии уравнения Лиувилля описьваются формулой

$$
V=\left(D+u_{x}\right) G\left(w, w_{x}, \ldots, w_{k_{1}}\right)+\left(\bar{D}+u_{y}\right) \bar{G}\left(\bar{w}, \bar{w}_{y}, \ldots, \bar{w}_{k_{2}}\right),
$$

где $w$ и $\bar{w}$ - минимальные $Y$ - и $X$ - интегралы, заданные формулой (2.42).

Отметим две особенности формулы (4.2). Во-первых, каждая симметрия является суммой двух симметрий, одна из которых зависит только от переменных $u, u_{x}, u_{x x}, \ldots$, а другая - от $u, u_{y}, u_{y y}, \ldots$. В несколько более слабой формулировке похожее утверждение для произвольного уравнения (1.2) доказано в [29].

Во-вторых, правая часть (4.2) содержит произвольные функции $G$ и $\bar{G}$. Далеко не при всяких функциях $G$ и $\bar{G}$ эволюционное уравнение $u_{\tau}=V$ является точно интегрируемым. Отметим, что запас высших симметрий для гиперболических уравнений типа sin-Gordon значительно скромнее: векторное пространство всех симметрий ограниченного порядка конечномерно. Зато в этом случае все высшие симметрии являются эволюционными уравнениями, интегрируемыми методом обратной задачи рассеяния.

Формула (4.2) обобщается на случай произвольного уравнения лиувиллевского типа. 
ТеОРема 5. Пусть для уравнения лиувиллевского типа (1.2) $H_{r}=H_{-s}=0$. Обозначим через $\mathscr{M}$ и $\overline{\mathscr{M}}$ дифференциальнье операторы

$$
\begin{aligned}
& \mathscr{M}=\bar{\psi} \frac{1}{H_{1}} D \frac{1}{H_{2}} \cdots D \frac{1}{H_{r-1}} D \frac{\psi H_{1} \cdots H_{r-1}}{\bar{\psi}}, \\
& \overline{\mathscr{M}}=\psi \frac{1}{H_{0}} \bar{D} \frac{1}{H_{-1}} \cdots \bar{D} \frac{1}{H_{1-s}} \bar{D} \frac{\bar{\psi} H_{0} \cdots H_{1-s}}{\psi} .
\end{aligned}
$$

Тогда для любых $Y-$ - X-интегралов $W$ и $\bar{W}$ выражсение

$$
V=\mathscr{M}(W)+\overline{\mathscr{M}}(\bar{W})
$$

является симметрией уравнения (1.2).

ДоКАЗАТЕЛЬСТво. Функции $V_{r}=0$ и $V_{r-1}=\bar{\psi} H_{1} \cdots H_{r-1} W$ удовлетворяют системе (2.44). Поэтому функция $V$, построенная с помошњю формулы (2.11), удовлетворяет (2.18). Легко видеть, что $V=\mathscr{M}(W)$. Следовательно, $V=\mathscr{M}(W)$ является симметрией. По аналогичной причине симметрией является $V=\overline{\mathscr{M}}(\bar{W})$. Поскольку множество всех симметрий является векторным пространством, теорема доказана.

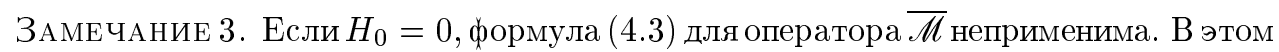
случае $\overline{\mathscr{M}}$ совпадает с оператором умножения на $\bar{\psi}$. Аналогично, если $H_{1}=0$, то $\mathscr{M}=\psi$.

Из (2.12) легко вьвести, что для всякой симметрии $V$

$$
V_{-s-1}=\psi W_{1}, \quad V_{r}=\bar{\psi} \bar{W}_{1},
$$

где $W_{1}$ и $\bar{W}_{1}$ - некоторые $Y$ - и $X$-интегралы. Согласно (2.43), (2.46) для симметрии, заданной формулой (4.4), $V_{-s-1}=\psi \mathscr{L}(W), V_{r}=\overline{\psi \mathscr{L}}(\bar{W})$. Мы видим, что если $V$ имеет вид (4.4), то $W_{1} \in \operatorname{Im} \mathscr{L}$ и $\bar{W}_{1} \in \operatorname{Im} \overline{\mathscr{L}}$. Последние условия нужно понимать следующим образом. Согласно предложению 3 всякий $Y$-интеграл является функцией от $x$, минимального $Y$-интеграла $w$ и его производных $w_{1}=D(w), w_{2}=D^{2}(w), \ldots$ Поскольку в силу теоремы 4 все коэффициенты оператора $\mathscr{L}$ являются $Y$-интегралами, этот оператор действует на множестве функций от переменных $x, w, w_{1}, w_{2}, \ldots$ Легко видеть, что не любая такая функция принадлежит $\operatorname{Im} \mathscr{L}$. Нетрудно показать, что условие $W_{1} \in \operatorname{Im} \mathscr{L}$ является необходимьм и достаточньм для того, чтобы симметрия задавалась формулой (4.4).

\section{§5. Уравнения лиувиллевского типа и преобразования Миуры для эволюционных уравнений}

В теории интегрируемых эволюционных уравнений хорошо известны (см., например, [37]-[39]) дифференциальные подстановки типа преобразования Миуры. Соотношение

$$
v=P\left(x, u, u_{x}, \ldots\right)
$$

называется дифференциальной подстановкой из уравнения

$$
u_{\tau}=f\left(x, u, u_{x}, \ldots\right)
$$


в уравнение

$$
v_{\tau}=g\left(x, v, v_{x}, \ldots\right),
$$

если для любого решения $u(x, \tau)$ первого уравнения функция (5.1) удовлетворяет второму. Далеко не для всякой функции $P\left(x, u, u_{x}, \ldots\right)$ можно найти пару эволюционных уравнений, которые бы связывала подстановка (5.1). С другой стороны, во всех известных примерах оказывается, если уж одна такая пара сушествует, то их бесконечно много.

Задача полного перечисления всех возможных дифференциальных подстановок является весьма сложной и в общей постановке не решена и по сей день. Однако тесная связь между дифференциальньми подстановками и гиперболическими уравнениями лиувиллевского типа, обнаруженная в [39], привела к серьезному прогрессу в этом вопросе.

Как мы увидим ниже, для всякого уравнения лиувиллевского типа его $Y$ - и $X$-интегралы минимального порядка задают дифференциальные подстановки вида (5.1). Вполне вероятно, что вообще все дифференциальные подстановки получаются из уравнений лиувиллевского типа с помощњю этой конструкции, но в настоящий момент мы совершенно не представляем, как бы это можно было доказать.

Сначала рассмотрим следуюшее уравнение лиувиллевского типа:

$$
u_{x y}=u u_{y}
$$

Легко проверить, что для него

$$
\psi=1, \quad \bar{\psi}=u_{y}, \quad H_{0}=0, \quad H_{1}=H_{2}=u_{y}, \quad H_{3}=0 .
$$

Минимальные $Y$ - и $X$-интегралы задаются формулой

$$
w=u_{x}-\frac{1}{2} u^{2}, \quad \bar{w}=\frac{u_{y y y}}{u_{y}}-\frac{3}{2} \frac{u_{y y}^{2}}{u_{y}^{2}} .
$$

Формулы (4.3), (2.40) с учетом замечания 3 дают

$$
\mathscr{M}=D^{2}+u D+u_{x}, \quad \overline{\mathscr{M}}=u_{y}, \quad \mathscr{L}=D^{3}+2 w D+w_{x} .
$$

Симметрии уравнения (5.2)

$$
u_{\tau}=\mathscr{M} w=u_{x x x}-\frac{3}{2} u^{2} u_{x}
$$

$$
u_{\tau}=\overline{\mathscr{M}} \bar{w}=u_{y y y}-\frac{3}{2} \frac{u_{y y}^{2}}{u_{y}}
$$

являются хорошо известными точно интегрируемыми эволюционньми уравнениями. Нетрудно проверить, что производная $w_{\tau}$, вычисленная в силу уравнения $(5.5)$, совпадает с $w_{x x}+3 w w_{x}$. Другими словами, $Y$-интеграл (5.3) уравнения (5.2) задает дифференциальную подстановку из уравнения мКд $\Phi$ (5.5) в уравнение Кортевега-де $\Phi$ риза. Эта подстановка - не что иное, как знаменитое преобразование Миуры. 
Аналогично, $X$-интеграл задает подстановку из уравнения (5.6) в уравнение $\mathrm{K}$ д $\Phi$ $\bar{w}_{\tau}=\bar{w}_{y y y}+3 \overline{w w}_{y}$.

Более того, для любой симметрии уравнения (5.2) вида

$$
u_{\tau}=\left(D^{2}+u D+u_{x}\right) G\left(x, w, w_{x}, \ldots, w_{n}\right)
$$

$Y$-интеграл $w=u_{x}-\frac{1}{2} u^{2}$ удовлетворяет некоторому эволюционному уравнению $w_{\tau}=Q\left(x, w, w_{x}, \ldots\right)$. Дело в том, что дифференцирование $D_{\tau}$ в силу уравнения $(5.7)$ коммутирует с $\bar{D}$ и поэтому переводит $Y$-интегралы снова в $Y$-интегралы. В частности, $Y$-интегралом является $D_{\tau}(w)$. Согласно предложению 1 , всякий $Y$-интеграл может быть представлен в виде (2.41). Поэтому $D_{\tau}(w)=Q\left(x, w, w_{x}, \ldots\right)$.

Уточним явньй вид функции $Q$ :

$$
\begin{aligned}
w_{\tau} & =(D-u) u_{\tau}=(D-u)\left(D^{2}+u D+u_{x}\right) G\left(x, w, w_{x}, \ldots, w_{n}\right) \\
& =\left(D^{3}+2 w D+w_{x}\right) G\left(x, w, w_{x}, \ldots, w_{n}\right)=\mathscr{L} G\left(x, w, w_{x}, \ldots, w_{n}\right) .
\end{aligned}
$$

Аналогичные рассуждения доказьвают следующее

ПРЕДЛОЖЕНИЕ 4. ДЛя любого уравнения лиувиллевского типа всякая симметрия вида

$$
u_{\tau}=\mathscr{M} G\left(x, w, w_{x}, \ldots\right),
$$

где $w$-минимальный $Y$-интеграл, связана дифференииальной подстановкой

$$
v=w\left(x, u, u_{x}, \ldots, u_{k}\right)
$$

с некоторым эволючионным уравнением вида

$$
v_{\tau}=Q\left(x, v, v_{x}, \ldots\right)
$$

Допустимая свобода $w \rightarrow f(x, w)$ в вьцборе минимального интеграла соответствует точечному преобразованию в уравнении (5.10).

Легко видеть, что правая часть $Q$ уравнения (5.10) имеет вид

$$
Q=w^{*} \mathscr{M}\left(G\left(x, v, v_{x}, \ldots\right)\right) \text {. }
$$

Здесь

$$
w^{*} \stackrel{\text { def }}{=} \sum_{i=0}^{k} \frac{\partial w}{\partial u_{i}} D^{i}
$$

- производная $\Phi$ реше от минимального $Y$-интеграла $w$. Поскольку $D_{\tau}(w)$ является $Y$-интегралом для произвольной функции $G$, все коэффициенты дифференциального оператора $w^{*} \mathscr{M}$ представляют собой $Y$-интегралы. 
ЗАмечАниЕ 4 . Во всех известных нам примерах оператор $w^{*} \mathscr{M}$ совпадает (при согласованном определении $w$ и $\psi$ ) с оператором $\mathscr{L}$, задаваемым формулой (2.43). Если это так, то согласно (2.43), (4.3) должна иметь место формула

$$
w^{*}=\frac{\bar{\psi}}{\psi} H_{0} H_{-1} \cdots H_{1-s} D \frac{1}{H_{1-s}} D \cdots \frac{1}{H_{0}} D \frac{1}{\bar{\psi}} .
$$

При предположении, что порядок $k$ минимального интеграла равен ord $\mathscr{L}-\operatorname{ord} \mathscr{M}$, мы умеем этот факт строго доказьвать.

Пусть у нас имеется произвольная дифференциальная подстановка первого порядка

$$
v=P\left(x, u, u_{x}\right)
$$

Сопоставим ей гиперболическое уравнение

$$
u_{x y}=-\frac{P_{u}}{P_{u_{x}}} u_{y}
$$

для которого функция $P$ является $Y$-интегралом. Для преобразования Миуры это уравнение совпадает с (5.2). Из (2.21) следует, что $H_{0}=0$ для любой функции $P$. Удивительно, что для всех известных примеров подстановок (5.13) уравнение (5.14) является уравнением лиувиллевского типа (т.е. $H_{r}=0$ для некоторого $\left.r>0\right)$.

Например, известной дифференциальной подстановке $v=u_{x}+\exp (u)+\exp (-u)$ соответствует уравнение

$$
u_{x y}=(\exp (-u)-\exp (u)) u_{y}
$$

для которого $H_{3}=0$.

ТЕОРЕМА 6. Уравнение (5.14) является уравнением лиувиллевского типа тогда и только тогда, когда существует замена $u \rightarrow f(x, u)$, после которой функчия $P\left(x, u, u_{x}\right)$ для полученного уравнения удовлетворяет соотношению вида

$$
u_{x}=\alpha(x, P) u^{2}+\beta(x, P) u+\gamma(x, P)
$$

для некоторых $\alpha, \beta$ и $\gamma$. При такой нормировке уравнение имеет (не обязательно минимальньй) $X$-интеграл вида

$$
\bar{W}=\frac{u_{y y y}}{u_{y}}-\frac{3}{2} \frac{u_{y y}^{2}}{u_{y}^{2}} .
$$

Итак, существует семейство подстановок вида (5.13), зависящее от трех произвольных функций $\alpha, \beta$ и $\gamma$. По-видимому, этими подстановками с точностью до замен $u \rightarrow f(x, u)$ и исчерпьвается список всех дифференциальных подстановок первого порядка. В частности, для (5.15) после замены $u \rightarrow \ln u$ функция $P$ удовлетворяет уравнению $u_{x}=-u^{2}+P u-1$. Для замены Коула-Хопфа $v=\frac{u_{x}}{u}$, линеаризуюшей уравнение Бюргерса $v_{\tau}=v_{x x}+2 v v_{x}$, имеем $u_{x}=P u$, и т. д. 


\section{§6. Список уравнений лиувиллевского типа}

6.1. Список. В этом разделе мы приводим полный с точностью до инволюции $x \leftrightarrow y$ и (вообще говоря, комплексных) преобразований вида

$$
x \rightarrow \zeta(x), \quad y \rightarrow \xi(y), \quad u \rightarrow \theta(x, y, u)
$$

список нелинейных уравнений лиувиллевского типа и их $Y$ - и $X$-интегралов.

КлАсс 1. Уравнения этого класса имеют вид

$$
u_{x y}=-\frac{W_{y}}{W_{u_{x}}}
$$

где функщия $W\left(x, y, u_{x}\right)$ определяется из уравнения вида

$$
u_{x}=q_{0}(x, y)+\sum_{i=1}^{n} \alpha_{i}(y) q_{i}(x, W),
$$

$\alpha_{i}, q_{i}$ - произвольные функщии. Функция $w=W\left(x, y, u_{x}\right)$ является $Y$-интегралом. Обозначим через

$$
L=\frac{\partial^{n}}{\partial y^{n}}+\sum_{i=0}^{n-1} c_{i}(y) \frac{\partial^{i}}{\partial y^{i}}
$$

однозначно определенный дифференциальньй оператор с ядром, порожденным $\alpha_{1}(y)$, $\alpha_{2}(y), \ldots, \alpha_{n}(y)$. Тогда $X$-интеграл (не обязательно минимальньй!) имеет вид

$$
\bar{w}=\bar{u}_{n}+\sum_{i=0}^{n-1} c_{i}(y) \bar{u}_{i}+\alpha(x, y),
$$

где $c_{i}(y)$ - коэффициенты оператора $L$. Функция $\alpha(x, y)$ определяется из соотношения

$$
\frac{\partial \alpha}{\partial x}=-L\left(q_{0}\right)
$$

КлАСС 2. (См. 5 5.)

$$
u_{x y}=-\frac{P_{u}}{P_{u_{x}}} u_{y}
$$

где

$$
u_{x}=\alpha(x, P) u^{2}+\beta(x, P) u+\gamma(x, P),
$$

$\alpha, \beta, \gamma-$ произвольные функции. Интегралами являются $w=P\left(x, u, u_{x}\right)$ и

$$
\bar{w}=\frac{\bar{u}_{3}}{\bar{u}_{1}}-\frac{3}{2} \frac{\bar{u}_{2}^{2}}{\bar{u}_{1}^{2}} .
$$

Хорошо известным примером уравнения из класса 2 является УРАВНЕНИЕ 1.

$$
u_{x y}=e^{u} u_{y}
$$


КлАСС 3.

$$
u_{x y}=A_{n}(x, y) \sqrt{u_{x} u_{y}},
$$

где $A_{n}(x, y)$ - любое решение уравнения

$$
h_{n}=0, \quad n \geqslant 1,
$$

левая часть которого $h_{n}$ определяется рекуррентными формулами

$$
\begin{gathered}
h_{k+1}=2 h_{k}-h_{k-1}-\frac{\partial^{2}}{\partial x \partial y} \ln h_{k}, \quad k=0,1, \ldots, n-2, \\
h_{0}=\frac{1}{4} A_{n}^{2}, \quad h_{1}=\frac{1}{4} A_{n}^{2}-\frac{\partial^{2}}{\partial x \partial y} \ln A_{n} .
\end{gathered}
$$

В частности, в этом классе содержатся все уравнения с

$$
A_{n}=\frac{2 n \lambda}{\lambda(x+y)-x y}
$$

и их вырождения с

$$
A_{n}=\frac{2 n}{x+y} .
$$

$Y$-интеграл $w=w\left(x, y, u, u_{1}, \ldots, u_{n}\right)$ имеет вид

$$
w=\frac{1}{A_{n}}\left(D-D\left(h_{1}\right)\right) \cdots\left(D-D\left(h_{1} \cdots h_{n-1}\right)\right) A_{n} \sqrt{u_{x}} .
$$

Интеграл $\bar{w}$ получается из него заменой $D \rightarrow \bar{D}, u_{x} \rightarrow u_{y}$.

УРАВНЕНИЕ 2.

$$
u_{x y}=e^{u} .
$$

Минимальные интегралы имеют вид

$$
w=u_{2}-\frac{1}{2} u_{1}^{2}, \quad \bar{w}=\bar{u}_{2}-\frac{1}{2} \bar{u}_{1}^{2} .
$$

УРАВНЕНИЕ 3.

$$
u_{x y}=\left(\frac{1}{u-x}+\frac{1}{u-y}\right) u_{x} u_{y} .
$$

Минимальные интегралы:

$$
w=\frac{u_{2}}{u_{1}}+\frac{1-2 u_{1}}{u-x}, \quad \bar{w}=\frac{\bar{u}_{2}}{\bar{u}_{1}}+\frac{1-2 \bar{u}_{1}}{u-y} .
$$

УРАВНЕНИЕ 4.

$$
u_{x y}=f(u) b\left(u_{x}\right)
$$

где

$$
f f^{\prime \prime}-f^{\prime 2}=0, \quad b b^{\prime}+u_{1}=0 .
$$


Минимальные интегралы задаются формулами

$$
w=\frac{u_{2}}{b}+f^{\prime} \frac{b}{f}, \quad \bar{w}=\bar{u}_{2}-\frac{f^{\prime}}{2 f} \bar{u}_{1}^{2}+\int f^{2} d u .
$$

УРАВНЕНИЕ 5.

$$
u_{x y}=f(u) b\left(u_{x}\right) \bar{b}\left(u_{y}\right)
$$

где

$$
(\ln f)^{\prime \prime}-f^{2}=0, \quad b b^{\prime}+u_{1}=0, \quad \bar{b} \bar{b}^{\prime}+\bar{u}_{1}=0 .
$$

Минимальные интегралы задаются формулами

$$
w=\frac{u_{2}}{b}+f^{\prime} \frac{b}{f}, \quad \bar{w}=\frac{\bar{u}_{2}}{\bar{b}}+f^{\prime} \frac{\bar{b}}{f} .
$$

УРАВНЕНИЕ 6.

$$
u_{x y}=\frac{1}{u} b\left(u_{x}\right) \bar{b}\left(u_{y}\right)
$$

где

$$
b b^{\prime}+c b+u_{1}=0, \quad \bar{b} \bar{b}^{\prime}+c \bar{b}+\bar{u}_{1}=0,
$$

где $c$ - произвольная постоянная. Минимальные интегралы:

$$
w=\frac{u_{2}}{b}-\frac{b}{u}, \quad \bar{w}=\frac{\bar{u}_{2}}{\bar{b}}-\frac{\bar{b}}{u} .
$$

УРАВНЕНИЕ 7.

$$
u_{x y}=\frac{1}{(x+y) b\left(u_{1}\right) \bar{b}\left(\bar{u}_{1}\right)}
$$

где

$$
b^{\prime}=b^{3}+b^{2}, \quad \bar{b}^{\prime}=\bar{b}^{3}+\bar{b}^{2}
$$

Минимальные интегралы:

$$
w=b u_{2}-\frac{1}{b(x+y)}, \quad \bar{w}=\bar{b} \bar{u}_{2}+\frac{1}{\bar{b}(x+y)} .
$$

УРАВНЕНИЕ 8.

$$
u_{x y}=\frac{1}{u} b\left(u_{x}\right) \bar{b}\left(u_{y}\right)
$$

где

$$
b b^{\prime}+b-2 u_{1}=0, \quad \bar{b} \bar{b}^{\prime}+\bar{b}-2 \bar{u}_{1}=0 .
$$

Минимальные интегралы:

$$
\begin{aligned}
& w=\frac{u_{3}}{b}+\frac{2\left(b-u_{1}\right)}{b^{3}} u_{2}^{2}+\frac{2\left(2 u_{1}+b\right)}{u b} u_{2}+\frac{b\left(u_{1}+b\right)}{u^{2}} \\
& \bar{w}=\frac{\bar{u}_{3}}{\bar{b}}+\frac{2\left(\bar{b}-\bar{u}_{1}\right)}{\bar{b}^{3}} \bar{u}_{2}^{2}+\frac{2\left(2 \bar{u}_{1}+\bar{b}\right)}{u \bar{b}} \bar{u}_{2}+\frac{\bar{b}\left(\bar{u}_{1}+\bar{b}\right)}{u^{2}} .
\end{aligned}
$$


УРАВНЕНИЕ 9.

$$
u_{x y}=\frac{1}{B_{u_{1}} \bar{B}_{\bar{u}_{1}}}\left[B \bar{B}\left(\frac{1}{6 u+x}+\frac{1}{6 u+y}\right)-\frac{1}{6 u+y} B-\frac{1}{6 u+x} \bar{B}\right]
$$

где

$$
\frac{1}{3} B^{3}-\frac{1}{2} B^{2}=u_{1}, \quad \frac{1}{3} \bar{B}^{3}-\frac{1}{2} \bar{B}^{2}=\bar{u}_{1} .
$$

Минимальные интегралы:

$$
\begin{aligned}
w= & D\left\{\ln \left[u_{2}-\frac{B^{4}(B-1)^{2}}{6 u+y}-\frac{B^{2}(B-1)^{4}}{6 u+x}\right]-\ln B(B-1)\right\} \\
& -\left[\left(\frac{1}{6 u+y}+\frac{1}{6 u+x}\right) B-\frac{1}{6 u+x}\right] B(B-1), \\
\bar{w}=\bar{D} & \left\{\ln \left[\bar{u}_{2}-\frac{\bar{B}^{4}(\bar{B}-1)^{2}}{6 u+x}-\frac{\bar{B}^{2}(\bar{B}-1)^{4}}{6 u+y}\right]-\ln \bar{B}(\bar{B}-1)\right\} \\
& -\left[\left(\frac{1}{6 u+y}+\frac{1}{6 u+x}\right) B-\frac{1}{6 u+x}\right] B(B-1) .
\end{aligned}
$$

6.2. Комментарии. 1 . Отметим, что для уравнений из класса 3 из формул (6.5) следует, что $\frac{\partial^{2}}{\partial x \partial y} \ln h_{1}=2 h_{1}-2 h_{0}$. Следовательно, соотношения (6.4), (6.5) и $h_{n}=0$ представляют собой открытую цепочку Тоды серии $C_{n}$, и поэтому все возможные функции $A_{n}$ могут быть найдены явно [40], [41].

2. Как видно из списка, для уравнений из класса 3 условие обрьва $H_{r}=H_{-s}=0$ может вьполняться при сколь угодно больших $r$ и $s$. Классификация уравнений с большими $r$ и $s$ доставляет значительные трудности. Основное наблюдение, позволяющее их преодолеть, опубликовано в [25].

3. Конечно, все дифференциальные уравнения для $f(u), b\left(u_{x}\right)$ и $\bar{b}\left(u_{y}\right)$ в случае уравнений $5-8$ легко интегрируются. Однако если приводить явные формулы, то из-за необходимости отдельно рассматривать частные решения размер списка увеличивается. Например, уравнение 4 эквивалентно одному из двух уравнений:

$$
u_{x y}=\exp (u) \sqrt{1-u_{x}^{2}}
$$

или

$$
u_{x y}=\sqrt{1-u_{x}^{2}}
$$

4. При приведении уравнений к одной из перечисленных выше канонических форм мы пользовались комплексными преобразованиями. Список вешественных канонических форм несколько шире.

5. Согласно известному наблюдению Гурса [42] уравнение вида

$$
u_{x y}=A(x, y) \sqrt{u_{x} u_{y}}
$$


подстановкой $v=\sqrt{u_{y}}$ сводится к линейному уравнению

$$
v_{x y}=\frac{A_{y}}{A} v_{x}+\frac{A^{2}}{4} v \text {. }
$$

Можно показать, что (6.6) является уравнением лиувиллевского типа тогда и только тогда, когда лиувиллевский тип имеет соответствующее уравнение (6.7).

Функции $h_{i}$ (см. описание уравнений из класса 3$)$ - это просто инварианты Лапласа уравнения (6.7). Всякое уравнение (6.7) лиувиллевского типа имеет $Y$-интегралы вида

$$
w=\sum_{i=0}^{m} M_{i}(x, y) \bar{v}_{i} .
$$

Соответствуюшие интегралы уравнения (6.6) могут быть получены из них подстановкой $v=\sqrt{u_{y}}$.

6. Грубо говоря, уравнения лиувиллевского типа - это уравнения, связанные различными подстановками с уравнением $v_{x y}=0$. Однако существуют нелинейные уравнения (1.2), связанные подстановками, скажем, с уравнением

$$
v_{x y}=v
$$

В частности, уравнение

$$
u_{x y}=2 \sqrt{u_{x} u_{y}}
$$

связано с (6.8) подстановкой

$$
v=c_{1} \sqrt{u_{x}}+c_{2} \sqrt{u_{y}}
$$

где $c_{i}$ - произвольные постоянные.

Как уравнение (6.8), так и уравнение (6.9) не являются уравнениями лиувиллевского типа. Таким образом, понятие "линеаризуемое уравнение" является более широким, чем понятие "уравнение лиувиллевского типа".

7. Сушествуют примеры уравнений, у которых цепочка инвариантов Лапласа обрьвается только в одну сторону. Например, для уравнения

$$
u_{x y}=\exp (u) b\left(u_{x}\right), \quad\left(u_{x}-b\right)\left(b+2 u_{x}\right)^{2}=1
$$

инвариант $H_{-1}$ равен нулю, в то время как $H_{i} \neq 0$ при $i>0$. Мы не знаем, является ли это уравнение интегрируемым в каком-либо смысле.

\section{§ 7. Заключительные замечания}

7.1. Об одном свойстве оператора $\mathscr{L}$. Рассмотрим множество $\mathscr{G}$ всех функций, зависяших от переменных

$$
x, \quad u_{0}=u, \quad u_{1}=u_{x}, \quad u_{2}=u_{x x}, \ldots .
$$


Для каждой функции $f\left(x, u, u_{1}, \ldots, u_{n}\right)$ положим

$$
f^{*} \stackrel{\text { def }}{=} \sum_{i=0}^{n} \frac{\partial f}{\partial u_{i}} D^{i} .
$$

Скобка Ли

$$
[f, g] \stackrel{\text { def }}{=} g^{*}(f)-f^{*}(g)
$$

задает на $\mathscr{G}$ структуру алгебры Ли. Скобка (7.1) соответствует коммутатору потоков эволющионных уравнений $w_{t_{1}}=f$ и $w_{t_{2}}=g$. Поэтому множество симметрий вида

$$
u_{\tau}=S\left(x, u, u_{1}, u_{2}, \ldots\right)
$$

данного уравнения лиувиллевского типа образует подалгебру Ли в $\mathscr{G}$ (как обычно, мы отождествляем симметрии с их правыми частями).

Оператор $\mathscr{L}=D^{3}+2 u D+u_{1}$, связаный с уравнением Лиувилля (см. пример 1$)$, обладает следующим замечательным свойством. Его образ является подалгеброй Ли в $\mathscr{G}$. А именно, легко проверить, что

$$
[\mathscr{L}(f), \mathscr{L}(g)]=\mathscr{L}\left(D(f) g-D(g) f+g^{*} \mathscr{L}(f)-f^{*} \mathscr{L}(g)\right)
$$

Поскольку $\mathscr{L}$ инъективен, последняя формула задает на $\mathscr{G}$ новую скобку Ли:

$$
[f, g]_{1} \stackrel{\text { def }}{=} D(f) g-D(g) f+g^{*} \mathscr{L}(f)-f^{*} \mathscr{L}(g) .
$$

Для всех известных нам гиперболических уравнений лиувиллевского типа оператор $\mathscr{L}$, заданный формулой (2.43), обладает тем же свойством. Мы покажем, что в свете результатов $\S 5$ это вьплядит довольно естественно.

Обозначим через $\mathscr{G}_{w}$ алгебру Ли функций от переменных $x, w, w_{1}, \ldots$ со скобкой вида (7.1). Согласно теореме 4 и предложению 3 все коэффициенты оператора $\mathscr{L}$ являются функциями от $x, w, w_{1}, \ldots$, где $w$-минимальньй $Y$-интеграл. Таким образом, у нас имеется дифференциальньй оператор $\mathscr{L}: \mathscr{G}_{w} \rightarrow \mathscr{G}_{w}$. Покажем, что при некоторых предположениях $\operatorname{Im} \mathscr{L}$ является подалгеброй Ли в $\mathscr{G}_{w}$.

Рассмотрим множество $\mathscr{S}$ всех симметрий вида (5.8) заданного уравнения лиувиллевского типа. Для всех известных примеров $\mathscr{S}$ совпадает с множеством всех симметрий вида (7.2) и поэтому является подалгеброй Ли в $\mathscr{G}$ относительно скобки (7.1). Как было показано в $\S 5$, образ симметрии (5.8) при подстановке (5.9) - это уравнение $w_{\tau}=Q$, где

$$
Q=w^{*} \mathscr{M}\left(G\left(x, w, w_{1}, \ldots\right)\right) .
$$

Поэтому множество всех функций вида (7.4) образует подалгебру относительно скобки (7.1). Чтобы закончить рассуждение, напомним, что согласно замечанию 4 $w^{*} \mathscr{M}=\mathscr{L}$ для всех известных уравнений лиувиллевского типа. 
Для заданного дифференциального оператора $\mathscr{L}$ с коэффициентами из $\mathscr{G}_{w}$ не очень трудно проверить, является ли его образ подалгеброй Ли. Это условие является довольно жестким.

Рассмотрим, например, операторы, “похожие” на (3.41). А именно, коэффициенты оператора (3.41) являются многочленами от $w, w_{1}, w_{2}, \ldots$ Сам оператор однороден, если считать, что $D$ имеет вес 1 , а вес $w_{i}$ равен $i+1$. Ниже приведен полный список операторов $\mathscr{L}$ порядков 2-6 указанной однородности таких, что $\operatorname{Im} \mathscr{L}$ - подалгебра Ли в $\mathscr{G}_{w}$. Оказалось, что все они раскладываются на множители первого порядка.

Операторы 2-го порядка:

$$
\mathscr{L}_{1}^{(2)}=D(D+w)
$$

Операторы 3-го порядка:

$$
\mathscr{L}_{1}^{(3)}=D(D+w)(D+w)
$$

Операторы 4-го порядка:

$$
\begin{aligned}
& \mathscr{L}_{1}^{(4)}=D(D+w)(D+w)(D+w), \\
& \mathscr{L}_{2}^{(4)}=D(D+w)(D+w)(D+2 w) .
\end{aligned}
$$

Операторы 5-го порядка:

$$
\begin{aligned}
& \mathscr{L}_{1}^{(5)}=D(D+w)(D+w)(D+w)(D+w), \\
& \mathscr{L}_{2}^{(5)}=D(D+w)(D+w)(D+2 w)(D+3 w) .
\end{aligned}
$$

Операторы 6-го порядка:

$$
\begin{aligned}
& \mathscr{L}_{1}^{(6)}=D(D+w)(D+w)(D+w)(D+w)(D+w), \\
& \mathscr{L}_{2}^{(6)}=D(D+w)(D+w)(D+w)(D+w)(D+2 w), \\
& \mathscr{L}_{3}^{(6)}=D(D+w)(D+w)(D+2 w)(D+3 w)(D+3 w), \\
& \mathscr{L}_{4}^{(6)}=D(D+w)(D+w)(D+2 w)(D+3 w)(D+4 w) .
\end{aligned}
$$

Отметим, что оператор $\mathscr{L}_{2}^{(5)}$ совпадает с (3.41). Интересно было бы найти все такие однородные операторы порядков больше шести и понять, что за красивая математика скрьвается за наборами натуральных коэффициентов, содержащихся в факторах операторов $\mathscr{L}_{i}^{(j)}$.

Можно показать, что для всякого гамильтонова дифференциального оператора (см. [43]) его образ - подалгебра в $\mathscr{G}$. Поэтому условие, рассмотренное выше, приводит к некоторому некососимметрическому обобшению понятия гамильтонова оператора. 
7.2. Системы лиувиллевского типа. Непосредственным обобщением уравнения Лиувилля на случай систем вида (1.5) являются открытые цепочки Тоды [41], [30], связанные с матрицами Картана простых алгебр Ли. Одна из эквивалентных форм записи этих систем имеет вид

$$
\left(u_{i}\right)_{x y}=\sum_{j} A_{i}^{j} \exp \left(u_{j}\right)
$$

где $A_{i}^{j}$ - элементы матрицы Картана соответствующей простой алгебры Ли. Хорошо известно, что эти системы обладают $Y$ - и $X$-интегралами (см. [44]).

Очевидно, что бо́льшую часть приведенных утверждений и конструкций этой статьи, связанных с уравнениями лиувиллевского типа, можно обобщить на случай систем (1.5). Однако здесь имеется следующая серьезная проблема, связанная с определением инвариантов Лапласа.

Оператор линеаризации (2.18) в этом случае - это оператор вида $D \bar{D}+a D+b \bar{D}+c$ с матричньми коэффициентами. Прямолинейное обобщение всех определений на матричньй случай (см. [45]) состоит в следующем. Главные инварианты Лапласа определяются формулами

$$
H_{1}=D(a)+b a-c, \quad H_{0}=\bar{D}(b)+a b-c,
$$

а матрицы $H_{i}$ при $i>1$ находятся рекуррентно из системы

$$
\begin{gathered}
\bar{D} H_{i}-H_{i} a_{i-1}+a_{i} H_{i}=0, \\
H_{i+1}=2 H_{i}+D\left(a_{i}-a_{i-1}\right)+\left[b, a_{i}-a_{i-1}\right]-H_{i-1},
\end{gathered}
$$

где $a_{0}=a$. Конечно, в скалярном случае эти формулы совпадают с соответствуюшими формулами из системы (2.6).

Если $H_{i}$ при $i \leqslant k$ и $a_{i}$ при $i \leqslant k-1$ уже известны, то из уравнения (7.5) определяется $a_{k}$, а затем из уравнения $(7.6)-H_{k+1}$. Однако если $\operatorname{det} H_{k}=0$, то $a_{k}$ либо вообще не существует, либо определяется не единственньм образом, а с точностью до матришы $\alpha$ такой, что $\alpha H_{k}=0$. При этом сушествование и свойства последуюших инвариантов Лапласа сушественно зависят от выбора $\alpha$.

Вырождение $\operatorname{det} H_{k}=0$ при некотором $k$ типично для открытых цепочек Тоды.

ПримеР 5. Рассмотрим цепочку Тоды серии $A_{2}$ :

$$
u_{x y}=-2 \exp u+\exp v, \quad v_{x y}=\exp u-2 \exp v .
$$

Оператор линеаризации имеет вид

$$
D \bar{D}+\left(\begin{array}{cc}
2 \exp u & -\exp v \\
-\exp u & 2 \exp v
\end{array}\right)
$$


Из формул (7.5), (7.6) находим

$$
\begin{gathered}
a_{1}=\frac{1}{3}\left(\begin{array}{cc}
-4 u_{y}+v_{y} & -2 u_{y}+2 v_{y} \\
2 u_{y}-2 v_{y} & u_{y}-4 v_{y}
\end{array}\right), \\
H_{2}=\left(\begin{array}{cc}
\exp u-2 \exp v & 2 \exp u-\exp v \\
-\exp u+2 \exp v & -2 \exp u+\exp v
\end{array}\right) .
\end{gathered}
$$

Мы видим, что $\operatorname{det} H_{2}=0$.

Нетрудно доказать [45] следующее общее утверждение (ср. с теоремой 3).

Теорема 7. Если система (1.5) обладает $Y$ - и X-интегралами

$$
W\left(x, y, \vec{u}, \vec{u}_{1}, \ldots, \vec{u}_{p}\right), \quad \bar{W}\left(x, y, \vec{u}, \overrightarrow{\vec{u}}_{1}, \ldots, \overline{\vec{u}}_{\bar{p}}\right),
$$

mо $\operatorname{det} H_{r}=\operatorname{det} H_{-s}=0$ для некоторых $r \leqslant p, s \leqslant \bar{p}-1$.

Таким образом, для систем типа цепочек Тоды некоторые из инвариантов Лапласа обязательно вырождены, и мы сталкиваемся с проблемой корректного определения последовательности инвариантов Лапласа.

Положим

$$
Z_{k}=H_{k} H_{k-1} \cdots H_{1}
$$

Из соотношений (7.5) следует, что

$$
Z_{k}(\bar{D}+a)=\left(\bar{D}+a_{k}\right) Z_{k} .
$$

Именно последовательность $Z_{k}$ (а не $H_{k}$ ) корректно определена для известных систем лиувиллевского типа. При этом если $H_{i}$ (а вместе с ними и $Z_{i}$ ) при $i \leqslant k$ и $a_{i}$ при $i \leqslant k-1$ уже известны, то $a_{k}$ следует определять из соотношения (7.8), а затем из уравнения

$$
H_{k+1}=(D+b) a_{k}-\left(\bar{D}+a_{k}\right) b+H_{k},
$$

эквивалентного соотношению (7.6), находить $H_{k+1}$. Матрица $a_{k}$ определена с точностью до произвольной матрищы $\alpha$ такой, что $\alpha Z_{k}=0$.

ТЕОРема 8. Пусть $H_{i}$ при $i \leqslant k$ уже известны и при $i<k$ выполнены условия

$$
(\bar{D}+a)\left(\operatorname{ker} Z_{i}\right) \subset \operatorname{ker} Z_{i}
$$

$u$

$$
\left(D-b^{T}\right)\left(\operatorname{ker} Z_{i}^{T}\right) \subset \operatorname{ker} Z_{i}^{T} .
$$

Тогда для существования $a_{k}$ необходимо и достаточно виполнение условия (7.10) при $i=k$. При этом $Z_{k+1}$ не зависит от произвольной матрицы $\alpha$, фигурирующей в определении $a_{k}$, если и только если при $i=k$ выполнено условие (7.11). 
ИДЕЯ ДОКАЗАТЕЛЬСТВА. Из формулы (7.8) следует, что для существования матрицы $a_{k}$ необходимо выполнение условия (7.10) при $i=k$. Достаточность следует из теоремы Кронекера-Капелли. Необходимость условия (7.11) вытекает из (7.9).

ЗАмечАнИЕ 5. В случае $a=b=0$ условия (7.10), (7.11) вьполнены, если пространства ker $Z_{k}$ и $\operatorname{ker} Z_{k}^{T}$ допускают базисы, состояшие из постоянных векторов.

Пример 6. Для цепочки Тоды серии $A_{3}$

$$
\begin{aligned}
& \left(u_{1}\right)_{x y}=2 \exp u_{1}-\exp u_{2}, \\
& \left(u_{2}\right)_{x y}=-\exp u_{1}+2 \exp u_{2}-\exp u_{3}, \\
& \left(u_{3}\right)_{x y}=-\exp u_{2}+2 \exp u_{3},
\end{aligned}
$$

все матрищы $Z_{k}$ однозначно определены и $\operatorname{rank} Z_{k}=4-k$. В частности, $Z_{4}=0$. Вектор $e_{1}=(1,1,1)^{T}$ образует базис $\operatorname{ker} Z_{2}$. Базис пространства $\operatorname{ker} Z_{3}$ можно выбрать следующим образом: $e_{1}$ и $e_{2}=(1,0,-1)^{T}$. Базисами $\operatorname{ker} Z_{2}^{T}$ и $\operatorname{ker} Z_{3}^{T}$ являются $f_{1}=(3,4,3)^{T}$ и $f_{1}, f_{2}=(1,0,-1)^{T}$. Таким образом, в этом случае $\operatorname{ker} Z_{k}$ и $\operatorname{ker} Z_{k}^{T}$ допускают постоянные базисы и поэтому выполнены условия (7.10), (7.11).

ПримеР 7. Для цепочки Тоды серии $C_{3}$

$$
\begin{aligned}
& \left(u_{1}\right)_{x y}=2 \exp u_{1}-\exp u_{2}, \\
& \left(u_{2}\right)_{x y}=-\exp u_{1}+2 \exp u_{2}-\exp u_{3}, \\
& \left(u_{3}\right)_{x y}=-2 \exp u_{2}+2 \exp u_{3},
\end{aligned}
$$

матрицы $Z_{k}$ однозначно определены,

$\operatorname{rank} Z_{1}=3, \quad \operatorname{rank} Z_{2}=2, \quad \operatorname{rank} Z_{3}=2, \quad \operatorname{rank} Z_{4}=1, \quad \operatorname{rank} Z_{5}=1$ и $Z_{6}=0$.

Все пространства $\operatorname{ker} Z_{k}$ и $\operatorname{ker} Z_{k}^{T}$ допускают постоянные базисы.

ПримеР 8. В случае цепочки Тоды $D_{3}$

$$
\begin{aligned}
& \left(u_{1}\right)_{x y}=2 \exp u_{1}-\exp u_{2}-\exp u_{3}, \\
& \left(u_{2}\right)_{x y}=-\exp u_{1}+2 \exp u_{2}, \\
& \left(u_{3}\right)_{x y}=-\exp u_{1}+2 \exp u_{3}
\end{aligned}
$$

$\operatorname{rank} Z_{k}=4-k$. Все пространства $\operatorname{ker} Z_{k}$ и $\operatorname{ker} Z_{k}^{T}$ обладают постоянными базисами.

ЗАмЕЧАнИЕ 6 . Как мы видим из рассмотренных примеров, индексы $i$, при которых происходит падение ранга матриц $Z_{i}$, совпадают с показателями соответствуюшей простой алгебры Ли, а номер $h$, для которого $Z_{h}=0$, равен числу Кокстера.

Обрьв цепочки $Z_{i}$ может быть положен в основу определения систем (1.5) лиувиллевского типа. 
ОПРЕДЕЛЕНИЕ 5. Назовем (1.5) системой лиувиллевского типа, если вьполнены условия (7.10) и (7.11) и сушествуют $r \geqslant 1$ и $s \geqslant 0$ такие, что $Z_{r}=Z_{-s} \equiv 0$.

Используя это определение, проклассифицируем все цепочки лиувиллевского типа вида

$$
\begin{aligned}
& \left(u_{1}\right)_{x y}=2 \exp u_{1}+k_{1} \exp u_{2}, \\
& \left(u_{2}\right)_{x y}=k_{2} \exp u_{1}+2 \exp u_{2}
\end{aligned}
$$

с невырожденной $\left(k_{1} k_{2} \neq-4\right)$ недиагональной матрищей Картана.

Легко проверить, что при любых $k_{1}, k_{2} \operatorname{rank} Z_{1}=2, \operatorname{rank} Z_{2}=1$. Далее, $Z_{3}=0$ тогда и только тогда, когда $k_{1}=k_{2}=-1$ (цепочка Тоды $A_{2}$ ).

Пусть $Z_{3} \neq 0$, тогда условие (7.10) с $i=3$ вьполнено, если и только если $k_{1}=-1$ или $k_{2}=-1$. Без потери обшности положим $k_{1}=-1$. Тогда $Z_{4}=0$ тогда и только тогда, когда $k_{2}=-2$ (цепочка Тоды $C_{2}$ ).

Если $k_{2} \neq-2$, тогда $\operatorname{rank} Z_{5}=1$ и условие $(7.10)$ с $i=5$ выполнено, если и только если $k_{2}=-3$. В этом случае $Z_{6}=0$ (цепочка Тоды $G_{2}$ ).

Таким образом, мы доказали, что все системы (7.12) лиувиллевского типа исчерпьваются цепочками Тоды, соответствуюшими простым алгебрам Ли ранга 2.

7.3. Уравнения типа sin-Gordon. Кроме уравнений sin-Gordon

$$
u_{x y}=\sin u,
$$

Цицейки

$$
u_{x y}=\exp (u)+\exp (-2 u),
$$

а также уравнения (1.3), хорошо известно интегрируемое уравнение

$$
u_{x y}=S(u) \sqrt{1-u_{x}^{2}},
$$

где $S$ - любое решение обыкновенного дифференщиального уравнения вида

$$
S^{\prime \prime}+\lambda^{2} S=0,
$$

где $\lambda$ - некоторая постоянная. С точностью до (комплексных) сдвигов и растяжений в (7.13) имеется три разных решения этого уравнения:

- $S=\sin u$,

- $S=u$,

- $S=1$.

В первом случае уравнение (7.13) связано с уравнением $v_{x y}=\sin v$ дифференциальной подстановкой $v=\arcsin u_{x}+u$, во втором - подстановкой $v=\arcsin u_{x}$. В третьем случае (7.13) является уравнением лиувиллевского типа (см. уравнение 4 в списке $\S 6$ ) и сводится подстановкой $v=\arcsin u_{x}$ к волновому уравнению $v_{x y}=0$.

Ниже мы приводим еше два интегрируемых уравнения (1.2). Эти уравнения связаны дифференциальньми подстановками с уравнением Цицейки. 
Модифицированные уравнения Цицейки. Оказьвается, что рассмотренное в $§ 3$ уравнение (3.24) - это вырождение следующего (вероятно, нового) уравнения

$$
u_{x y}=S(u) b\left(u_{x}\right) \bar{b}\left(u_{y}\right)
$$

интегрируемого методом обратной задачи. Здесь функщии $b\left(u_{x}\right)$ и $\bar{b}\left(u_{y}\right)$ определены формулами

$$
\left(u_{x}-b\right)\left(b+2 u_{x}\right)^{2}=1, \quad\left(u_{y}-\bar{b}\right)\left(\bar{b}+2 u_{y}\right)^{2}=1,
$$

а $S$-произвольное решение дифференщиального уравнения

$$
S^{\prime \prime}-2 S S^{\prime}-4 S^{3}=0 .
$$

Отметим, что уравнение (7.15) имеет первьй интеграл

$$
I=\left(S^{\prime}-2 S^{2}\right)^{2}\left(S^{\prime}+S^{2}\right) .
$$

Как сообшил нам М. Павлов, подстановки $S=Q^{\prime}$ и $Q^{\prime 2}=F(Q)$ сводят (7.15) к линейному уравнению $F^{\prime \prime}-2 F^{\prime}-8 F=0$. Отсюда следует, что функция $Z=\exp (-2 Q)$ удовлетворяет уравнению $Z^{\prime 2}=c_{1} Z^{3}+c_{2}$, где $c_{i}$ - некоторые постоянные.

Частное решение $S=\frac{1}{u}$ приводит к уравнению (3.24). Другое решение $S=-\frac{1}{2 u}$ соответствует уравнению лиувиллевского типа

$$
u_{x y}=-\frac{1}{2 u} b\left(u_{x}\right) \bar{b}\left(u_{y}\right) .
$$

Последнее уравнение эквивалентно одному из семейства уравнений 6 (см. список 6 ).

Уравнение (7.14) связано с уравнением Цицейки

$$
v_{x y}=c_{1} \exp (v)+c_{2} \exp (-2 v)
$$

дифференциальной подстановкой

$$
v=-\frac{1}{2} \ln \left(u_{x}-b\right)-\frac{1}{2} \ln \left(u_{y}-\bar{b}\right)+P(u),
$$

где функщия $P(u)$ определяется из дифференщиального уравнения

$$
P^{\prime 2}-2 S P^{\prime}-3 S^{\prime}-2 S^{2}=0 .
$$

При этом константы $c_{1}$ и $c_{2}$ удовлетворяют соотношению $c_{1}^{2} c_{2}=-I / 27$.

Кроме уравнения (7.14), с уравнением Цицейки связано уравнение

$$
u_{x y}=S(u) b\left(u_{x}\right)
$$

где $S^{\prime \prime}=0$. С точностью до сдвигов и растяжений имеется два различных случая:

$$
\begin{aligned}
& \text { - } S=3 u, \\
& \text { - } S=1 \text {. }
\end{aligned}
$$

В первом случае (см. [46]) уравнение (7.16) связано с уравнением $v_{x y}=\exp (v)+$ $\exp (-2 v)$ дифференциальной подстановкой

$$
v=-\frac{1}{2} \ln \left(u_{x}-b\right)
$$

Эта подстановка обратима в смысле работы [46].

В втором случае (7.16) является уравнением лиувиллевского типа (принадлежит классу 1 в списке $\S 6$ ) и сводится подстановкой (7.17) к волновому уравнению $v_{x y}=0$. 
ГипотеЗА. Перечисленными в этом разделе уравнениями исчерпьвается, с точностью до инволюции $x \leftrightarrow y$ и (комплексных) преобразований (6.1), список уравнений (1.2), интегрируемых методом обратной задачи рассеяния.

В заключение заметим, что набор инвариантов Лапласа $H_{i}$ для уравнения лиувиллевского типа удовлетворяет системе (2.22) с условием обрьва $H_{r}=H_{-s}=0$. Эта система представляет собой одну из разновидностей открытой цепочки Тоды серии $A$, и поэтому сама является системой лиувиллевского типа.

Возникает вопрос, как обобщить это наблюдение на инварианты Лапласа на случай уравнений типа sin-Gordon. Наиболее естественное предположение о том, что инварианты для таких уравнений должны удовлетворять периодически замкнутым цепочкам Тоды, оказывается неверным. Было бы интересно найти описание уравнений типа sin-Gordon в терминах их инвариантов Лапласа.

\section{СПИСОК ЛИТЕРАТУРЫ}

[1] G. Darboux. Sur les équations aux dérivées partielles du second ordre // Ann. Sci. Ecole Norm. Sup. 1870. V. 7. P. 163-173.

[2] G. Darboux. Leçons sur la théorie générale des surfaces. V. II. Paris: Hermann, 1915.

[3] E. Goursat. Leçons sur l'intégration des équations aux dérivées partielles du second ordre à deux variables indépendantes. V. I, II. Paris: Hermann, 1896, 1898.

[4] Д. Ф. Егоров. Уравнения с частными производньми 2-го порядка по двум независимым переменным. Общая теория интегралов: характеристики // Ученые записки Императорского Моск. ун-та. 1899. Т. 15.

[5] E. Gau. Sur l'intégration des équations aux dérivées partielles du second ordre par la méthode de M. Darboux // J. Math. Pures Appl. 1911. V. 7. P. 123-240.

[6] E. Vessiot. Sur les équations aux dérivées partielles du second ordre, $F(x, y, p, q, r, s, t)=0$, intégrables par la méthode de Darboux // J. Math. Pures Appl. 1939. V. 18. P. 1-61.

[7] Ф. Трикоми. Лекции по уравнениям в частных производных. М.: ИЛ, 1957.

[8] В.Е. Захаров, С. В. Манаков, С.П. Новиков, Л. П. Питаевский. Теория солитонов: метод обратной задачи. М.: Наука, 1980.

[9] Л. А. Тахтаджян, Л. Д. Фаддеев. Гамильтонов подход в теории солитонов. М.: Наука, 1986.

[10] R. Gosse. La méthode de Darboux et les équations $s=f(x, y, z, p, q) \quad / /$ Mémorial de Sciences mathématiques, fasc. 12, Paris, 1926.

[11] E. Goursat. Sur les intégrales de l'équation $s=f(x, y, z, p, q) / /$ C. R. Acad. Sci. Paris. 1903. V. 136. P. 1383-1384.

[12] É. Lainé. Sur l'application de la méthode de Darboux aux equations $s=f(x, y, z, p, q) / /$ C. R. Acad. Sci. Paris. 1926. V. 182. P. 1127-1128.

[13] É. Lainé. Sur les équations $s=f(x, y, z, p, q)$ intégrables par la méthode de Darboux // C. R. Acad. Sci. Paris. 1928. V. 186. P. 209-210.

[14] D. H. Parsons. The extension of Darboux's method // Mémorial de Sciences Mathématiques, fasc. 142, Paris, 1960.

[15] М. Ю. Звягин. Уравнения второго порядка, приводимые преобразованием Бэклунда к $z_{x y}=0$ // Докл. АН СССР. 1991. Т. 316. №1. С. 36-40.

[16] А.Н. Лезнов, В. Г. Смирнов, А. Б. Шабат. Группа внутренних симметрий и условия интегрируемости двумерных динамических систем // ТМФ. 1982. Т. 51. № 1. С. 10-22.

[17] I. M. Anderson, N. Kamran. The variational bicomplex for second order scalar partial differential equations in the plane // Preprint. Montréal: Centre de Recherches Mathematiques, Université de Montréal, 1994.

[18] I. M. Anderson, N. Kamran. The variational bicomplex for hyperbolic second-order scalar partial differential equations in the plane // Duke Math. J. 1997. V. 87. № 2. P. 265-319. 
[19] А. В. ЖКибер, В. В. Соколов, С. Я. Старцев. О нелинейных гиперболических уравнениях, интегрируемых по Дарбу // Докл. РАН. 1995. Т. 343. № 6. С. 746-748.

[20] V. V. Sokolov, A. V. Zhiber. On the Darboux integrable hyperbolic equation // Phys. Lett. A. 1995. V. 208. P. 303-308.

[21] I. M. Anderson, M. Juras. Generalized Laplace invariants and the method of Darboux // Duke Math. J. 1997. V. 89. № 2. P. 351-375.

[22] А. В. ЖКибер, А. Б. Шабат. Уравнения Клейна-Гордона с нетривиалной группой // Докл. АН СССР. 1979. Т. 247. № 5. С. 1103-1107.

[23] А. В. ЖКибер, А. Б. Шабат. Системы уравнений $u_{x}=p(u, v), v_{y}=q(u, v)$, обладающие симметриями // Докл. АН СССР. 1984. Т. 277. №1. С. 29-33.

[24] А. Б. Борисов, С. А. Зыков. Одевающая цепочка дискретных симметрий и размножение нелинейных уравнений // ТМФ. 1998. Т. 115. № 2. С. 199-214.

[25] А. В. ЖКибер, В.В.Соколов. Новый пример гиперболического нелинейного уравнения, обладающего интегралами // ТМФ. 1999. Т. 120. №1. С. 20-26.

[26] J. Le Roux. Extensions de la méthode de Laplace aux équations linéaires aux derivées partielles d'ordre supérieur au second // Bull. Soc. Math. France. 1899. V. 27. P. 237-262.

[27] C. Athorne. A $\mathbf{Z}^{2} \times \mathbf{R}^{3}$ Toda system // Phys. Lett. A. 1995. V. 206. P. 162-166.

[28] С. П. Царев. Факторизация линейных дифференциальных операторов с частными производными и метод Дарбу интегрирования нелинейных уравнений с частными производными // ТМФ. 2000. Т. 122. №1. С. 144-160.

[29] А. В. ЖКибер. Квазилинейные гиперболические уравнения с бесконечной алгеброй симметрий // Изв. РАН. Сер. матем. 1994. Т. 58. № 4. С. 33-54.

[30] А. Б. Шабат, Р.И.Ямилов. Экспоненциальные системы типа І и матрицы Картана // Препринт. Уфа: БФАН СССР, 1981.

[31] А.В. ЖКибер, Н.Х. Ибрагимов, А.Б. Шабат. Уравнения типа Лиувилля // Докл. АН СССР. 1979. Т. 249. № 1. С. 26-29.

[32] А. Н. Лезнов, М. В. Савельев, В.Г. Смирнов. Общие решения двумерной системы уравнений Вольтерра, реализующих преобразования Беклунда для цепочки Тода // ТМФ. 1981. T. 42. № 2. C. $216-234$.

[33] V. V. Sokolov, A. B. Shabat. Classification of integrable evolution equations // Soviet Sci. Rev. Sect. C. Math. Phys. Rev. 1984. V. 4. P. 221-280.

[34] A.S. Fokas. Symmetries and integrability // Stud. Appl. Math. 1987. V. 77. P. 253-299.

[35] А. В. Михайлов, А. Б. Шабат, Р.И.Ямилов. Симметрийный подход к классифйкации нелинейных уравнений. Полные списки интегрируемых систем // УМН. 1987. Т. 42. № 4. C. $3-53$.

[36] A. V. Mikhailov, A. B. Shabat, V. V. Sokolov. The symmetry approach to classification of integrable equations // What is integrability? Berlin: Springer-Verlag, 1991. P. 115-184.

[37] R. M. Miura. Korteweg-de Vries equation and generalizations. I: A remarkable explicit nonlinear transformation // J. Math. Phys. 1968. V. 9. P. 1202-1204.

[38] С. И. Свинолупов, В.В. Соколов, Р. И. Ямилов. Преобразования Бэклунда для интегрируемых эволюционных уравнений // Докл. АН СССР. 1983. Т. 271. № 4. С. 802-805.

[39] В. В. Соколов. О симметриях эволюционных уравнений // УМН. 1988. Т. 43. №5. C. $133-163$.

[40] T. Moutard. Sur la construction des équations de la forme $\frac{1}{z} \frac{d^{2} z}{d x d y}=\lambda(x, y)$, qui admettent une intégrale générale explicite // J. École Polytech. 1878. V. 45. P. 1-11.

[41] А. Н. Лезнов, М. В. Савельев. Групповые методы интегрирования нелинейных динамических систем. М.: Наука, 1985.

[42] E. Goursat. Sur une transformation de l'équation $s^{2}=4 \lambda(x, y) p q / /$ Bull. Soc. Math. France. 1900. V. 28. P. 1-6.

[43] П. Олвер. Приложения групп Ли к дифференциальным уравнениям. М.: Мир, 1989.

[44] A. B. Shabat. Higher symmetries of two-dimensional lattices // Phys. Lett. A. 1995. V. 200. P. 121-133. 
[45] С. Я. Старцев. Инварианты Лапласа интегрируемых гиперболических уравнений и дифоференциальные подстановки типа преобразования Миуры // Дисс. ... канд. физ.-матем. наук. Уфа: ИМ РАН, 1997.

[46] V. V. Sokolov, S. I. Svinolupov. On nonclassical invertible transformations of hyperbolic equations // European J. Appl. Math. 1995. V. 6. P. 145-156.

Институт механики Уфимского научного центра РАН;

Центр нелинейных исследований при ИТФ им. Л. Д. Ландау РАН

Поступила в редакцию

20.08 .2000 